\title{
STATUS OF THE STANDARD MODEL AND BEYOND
}

\author{
G. Altarelli \\ TH Division, CERN, CH-1211 Geneva 23 and Univ. di Roma Tre, Rome, Italy \\ E-mail: iguido. altarelili@cern.chi
}

AbSTRACT: Precision tests support the Standard Model and give indirect evidence for a light, fundamental Higgs. Yet, we do not believe in the Standard Model, both for conceptual and phenomenological reasons. The experimental search for the Higgs and supersymmetry continues at LEP2, the Tevatron and the LHC.

\section{Introduction}

The running of LEP1 was terminated in 1995 and close-to-final results of the data analysis are now available. The LEP2 programme is under way and new tests of the Standard Model are being accomplished. LEP and SLC started in 1989 and the first results from the collider run at the Tevatron were also first presented at about that time. I went back to my rapporteur talk at the Stanford Conference in August 1989 and I found the following best values quoted there for some of the key quantities of interest for the Standard Model phenomenology: $m_{Z}=91120(160) \mathrm{MeV}$; $m_{t}=130(50) \mathrm{GeV} ; \sin ^{2} \theta_{\text {eff }}=0.23300(230)$ and $\alpha_{s}\left(m_{Z}\right)=0.110(10)$. Now, after nearly ten years of experimental and theoretical work (in particular with 16 million $\mathrm{Z}$ events analysed altogether by the four LEP experiments) the corresponding numbersare: $m_{Z}=91186.3(2.0) \mathrm{MeV} ; m_{t}=$ $173.8(5) \mathrm{GeV} ; \sin ^{2} \theta_{\text {eff }}=0.23155(19)$ and $\alpha_{s}\left(m_{Z}\right)$ $=0.119(3)$. Thus the progress is quite evident. The top quark has been at last found and the errors on $m_{Z}$ and $\sin ^{2} \theta_{\text {eff }}$ went down by two and one orders of magnitude respectively. At the start the goals of LEP, SLC and the Tevatron were: a) perform precision tests of the SM at the level of a few permil accuracy; b) count neutrinos $\left(N_{\nu}=2.994(11)\right)$; c) search for the top quark $\left(m_{t}=173.8(5) \mathrm{GeV}\right)$; d) search for the Higgs $\left(m_{H}>90 \mathrm{GeV}\right)$; e) search for new parti- cles (none found). While for most of the issues the results can be summarised in very few bits, as just shown, for the first one the task is by far more complex. We will review precision tests of the SM in the following.

The validity of the SM has been confirmed to a level that I can say was unexpected at the beginning. The impressive success of the SM poses strong limitations on the possible forms of new physics. Favoured are models of the Higgs sector and of new physics that preserve the SM structure and only very delicately improve it, as is the case for fundamental Higgs(es) and Supersymmetry. Disfavoured are models with a nearby strong non perturbative regime that almost inevitably would affect the radiative corrections, as for composite Higgs(es) or technicolor and its variants. In the second part of these lectures I will review the arguments that, in spite of the experimental success of the SM, predict that new physics, probably supersymmetry, will be found in the near future at present accelerators or at the LHC.

\section{Status of the Data on Precision Electroweak Tests}

The relevant electro-weak data together with their

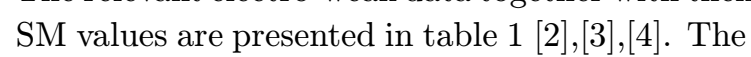


SM predictions correspond to a fit of all the available data (including the directly measured values of $m_{t}$ and $\left.m_{W}\right)$ in terms of $m_{t}, m_{H}$ and $\alpha_{s}\left(m_{Z}\right)$, described later in Section 3, Table 4.

Other important derived quantities are, for example, $N_{\nu}$ the number of light neutrinos, obtained from the invisible width: $N_{\nu}=2.994(11)$, which shows that only three fermion generations exist with $m_{\nu}<45 \mathrm{GeV}$. This is one of the most important results of LEP. Other important quantities are the leptonic width $\Gamma_{l}$, averaged over e, $\mu$ and $\tau: \Gamma_{l}=83.90(10) M e V$ and the hadronic width $\Gamma_{h}=1742.3(2.3) M e V$.

For indicative purposes, in Table 1 the "pulls" are also shown, defined as: pull $=($ data point- fit value)/(error on data point). At a glance we see that the agreement with the SM is quite good. The distribution of the pulls is statistically normal. The presence of a few $\sim 2 \sigma$ deviations is what is to be expected. However it is maybe worthwhile to give a closer look at these small discrepancies.

One persistent feature of the data is the difference between the values of $\sin ^{2} \theta_{\text {eff }}$ measured at LEP and at SLC (although the discrepancy is going down in the most recent data). The value of $\sin ^{2} \theta_{\text {eff }}$ is obtained from a set of combined asymmetries. From asymmetries one derives the ratio $x=g_{V}^{l} / g_{A}^{l}$ of the vector and axial vector couplings of the $Z$, averaged over the charged leptons. In turn $\sin ^{2} \theta_{\text {eff }}$ is defined by $x=1-4 \sin ^{2} \theta_{\text {eff }}$. SLD obtains $\mathrm{x}$ from the single measurement of $A_{L R}$, the left-right asymmetry, which requires longitudinally polarized beams. The distribution of the present measurements of $\sin ^{2} \theta_{\text {eff }}$ is shown in Fig. $i_{1}^{1}$. The LEP average, $\sin ^{2} \theta_{\text {eff }}=0.23187(24)$, differs by $2.2 \sigma$ from the SLD value $\sin ^{2} \theta_{\text {eff }}=0.23101(31)$. The most precise individual measurement at LEP is from $A_{b}^{F B}$ : the combined LEP error on this quantity is comparable to the SLD error, but the two values are $2.5 \sigma$ 's away. One might attribute this to the fact that the $\mathrm{b}$ measurement is more delicate and affected by a complicated systematics. In fact one notices from Fig. $\overline{1}_{1}^{1}$ that the value obtained at LEP from $A_{l}^{F B}$, the average for $\mathrm{l}=\mathrm{e}, \mu$ and $\tau$, is somewhat low (indeed quite in agree-

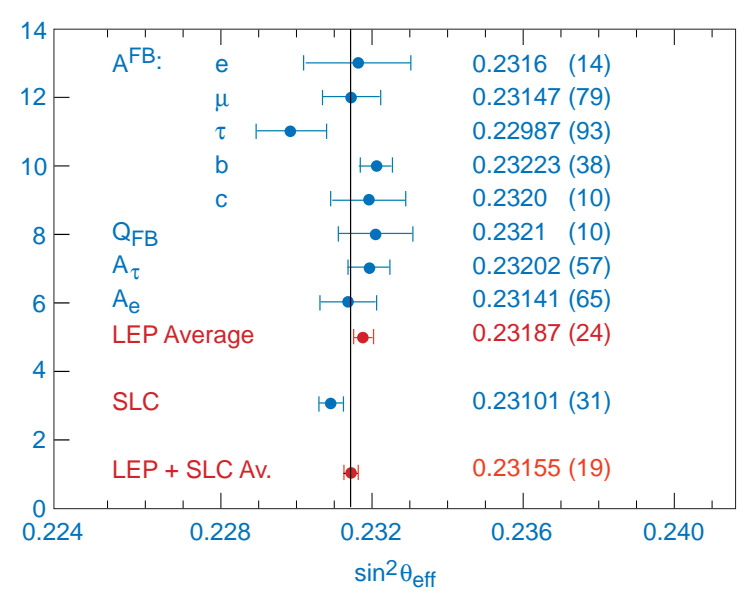

Figure 1: A summary of $\sin ^{2} \theta_{\text {eff }}$ measurements

ment with the SLD value). However the statement that LEP and SLD agree on leptons while they only disagree when the $\mathrm{b}$ quark is considered is not quite right. First, the low value of $\sin ^{2} \theta_{\text {eff }}$ found at LEP from $A_{l}^{F B}$ turns out to be entirely due to the $\tau$ lepton channel which leads to a central value different than that of e and $\mu$. The e and $\mu$ asymmetries, which are experimentally simpler, are perfectly on top of the SM fit. Second, if we take only e and $\mu$ asymmetries at LEP and disregard the $\mathrm{b}$ and $\tau$ measurements the LEP average becomes $\sin ^{2} \theta_{\text {eff }}=0.23168(36)$, which is still $1.4 \sigma$ away from the SLD value. Thus it is difficult to find a simple explanation for the SLD-LEP discrepancy on $\sin ^{2} \theta_{\text {eff }}$. In the following we will tentatively use the official average

$$
\sin ^{2} \theta_{\text {eff }}=0.23155 \pm 0.00019
$$

obtained by a simple combination of the LEPSLC data. One could be more conservative and enlarge the error because of the larger dispersion, but the difference would not be too large. Also, this dispersion has decreased in the most recent data. The data-taking by the SLD experiment is still in progress and also at LEP seizable improvements on $A_{\tau}$ and $A_{b}^{F B}$ are foreseen as soon as the corresponding analyses will be completed. We hope to see the difference to be further reduced in the end.

From the above discussion one may wonder if there is evidence for something special in the $\tau$ channel, or equivalently if lepton universality 
is really supported by the data. Indeed this is the case: the hint of a difference in $A_{\tau}^{F B}$ with respect to the corresponding e and $\mu$ asymmetries is not confirmed by the measurements of $A_{\tau}$ and $\Gamma_{\tau}$ which appear normal. In principle the fact that an anomaly shows up in $A_{\tau}^{F B}$ and not in $A_{\tau}$ and $\Gamma_{\tau}$ is not unconceivable because the FB lepton asymmetries are very small and very precisely measured. For example, the extraction of $A_{\tau}^{F B}$ from the data on the angular distribution of $\tau$ 's could be biased if the imaginary part of the continuum was altered by some non universal new physics effect. But a more trivial experimental problem is at the moment the most plausible option.

A similar question can be asked for the b couplings. We have seen that the measured value of $A_{b}^{F B}$ is $1.8 \sigma$ 's below the SM fit. At the same time $R_{b}$ which used to show a major discrepancy is now only about $1 \sigma$ 's away from the SM fit (as a result of the more sophisticated second generation experimental techniques). There is a $-2.5 \sigma$ deviation on the measured value of $A_{b}$ vs the SM expectation. That somewhat depends on how the data are combined. Let us discuss this point in detail. $A_{b}$ can be measured directly at SLC by taking advantage of the beam longitudinal polarization. The SLC value (see Table 1 ) is $2.2 \sigma^{\prime}$ s below the SM value. At LEP one measures $A_{b}^{F B}$ $=3 / 4 A_{e} A_{b}$. One can then derive $A_{b}$ by inserting a value for $A_{e}$. The question is what to use for $A_{e}$ : the LEP value obtained, using lepton universality, from the measurements of $A_{l}^{F B}, A_{\tau}, A_{e}$ : $A_{e}=0.1470(27)$, or the combination of LEP and SLD etc. Since we are here concerned with the b couplings it is perhaps wiser to obtain $A_{b}$ from LEP by using the SM value for $A_{e}$ (that is the pull-zero value of table 1): $A_{e}^{S M}=0.1467(15)$. With the value of $A_{b}$ derived in this way from LEP (which is $1.7 \sigma$ 's below the SM value) we finally obtain

$$
\begin{gathered}
A_{b}=0.890 \pm 0.018(\mathrm{LEP}+\mathrm{SLD}, \\
\left.A_{e}=A_{e}^{S M}:-2.5 \sigma\right)
\end{gathered}
$$

In the $\mathrm{SM} A_{b}$ is so close to 1 because the b quark is almost purely left-handed. $A_{b}$ only depends on the ratio $r=\left(g_{R} / g_{L}\right)^{2}$ which in the $\mathrm{SM}$ is small: $r \sim 0.033$. To adequately decrease $A_{b}$ from its $\mathrm{SM}$ value one must increase $\mathrm{r}$ by a factor of about 1.6, which appears large for a new physics effect. Also such a large change in $r$ must be compensated by decreasing $g_{L}^{2}$ by a small but fine-tuned amount in order to counterbalance the corresponding large positive shift in $R_{b}$. In view of this the most likely way out is that $A_{b}^{F B}$ and $A_{b}$ have been a bit underestimated at LEP and actually there is no anomaly in the b couplings. Then the LEP value of $\sin ^{2} \theta_{\text {eff }}$ would slightly move down, in the direction of decreasing the SLD-LEP discrepancy.

\section{Precision Electroweak Data and the Standard Model}

For the analysis of electroweak data in the SM one starts from the input parameters: some of them, $\alpha, G_{F}$ and $m_{Z}$, are very well measured, some other ones, $m_{f_{\text {light }}}, m_{t}$ and $\alpha_{s}\left(m_{Z}\right)$ are only approximately determined while $m_{H}$ is largely unknown. With respect to $m_{t}$ the situation has much improved since the CDF/D0 direct measurement of the top quark mass. From the input parameters one computes the radiative corrections to a sufficient precision to match the experimental capabilities. Then one compares the theoretical predictions and the data for the numerous observables which have been measured, checks the consistency of the theory and derives constraints on $m_{t}, \alpha_{s}\left(m_{Z}\right)$ and hopefully also on $m_{H}$.

Some comments on the least known of the input parameters are now in order. The only practically relevant terms where precise values of the light quark masses, $m_{f_{\text {light }}}$, are needed are those related to the hadronic contribution to the photon vacuum polarisation diagrams, that determine $\alpha\left(m_{Z}\right)$. This correction is of order $6 \%$, much larger than the accuracy of a few per mille of the precision tests. Fortunately, one can use the actual data to in principle solve the related ambiguity. But we shall see that the left over uncertainty is still one of the main sources of theoretical error. As is well known [ilin, the QED 


\begin{tabular}{|l|l|c|}
\hline Quantity & Data (August'98) & Pull \\
\hline$m_{Z}(\mathrm{GeV})$ & $91.1867(21)$ & 0.1 \\
$\Gamma_{Z}(\mathrm{GeV})$ & $2.4939(24)$ & -0.8 \\
$\sigma_{h}$ (nb) & $41.491(58)$ & 0.3 \\
$R_{h}$ & $20.765(26)$ & 0.7 \\
$R_{b}$ & $0.21656(74)$ & 0.9 \\
$R_{c}$ & $0.1735(44)$ & 0.3 \\
$A_{F B}^{l}$ & $0.01683(96)$ & 0.7 \\
$A_{\tau}$ & $0.1431(45)$ & -0.8 \\
$A_{e}$ & $0.1479(51)$ & 0.25 \\
$A_{F B}^{b}$ & $0.0990(21)$ & -1.8 \\
$A_{F B}^{c}$ & $0.0709(44)$ & -0.6 \\
$A_{b}$ (SLD direct) & $0.867(35)$ & -1.9 \\
$A_{c}$ (SLD direct) & $0.647(40)$ & -0.5 \\
$\sin ^{2} \theta_{\text {eff }}($ LEP-combined) & $0.23187(24)$ & 1.3 \\
$A_{L R} \rightarrow \sin { }^{2} \theta_{\text {eff }}$ & $0.23101(31)$ & -1.8 \\
$m_{W}$ (GeV) (LEP2+p $\left.\bar{p}\right)$ & $80.39(60)$ & -0.4 \\
$1-\frac{m_{W}^{2}}{m_{Z}^{2}}(\nu \mathrm{N})$ & $0.2253(21)$ & 1.1 \\
$Q_{W}$ (Atomic PV in Cs) & $-72.11(93)$ & 1.2 \\
$m_{t}$ (GeV) & $173.8(5.0)$ & 0.5 \\
\hline
\end{tabular}

Table 1: Data on precision electroweak tests

running coupling is given by:

$$
\begin{gathered}
\alpha(s)=\frac{\alpha}{1-\Delta \alpha(s)} \\
\Delta \alpha(s)=\Pi(s)=\Pi_{\gamma}(0)-\operatorname{Re}_{\gamma}(s)
\end{gathered}
$$

where $\Pi(s)$ is proportional to the sum of all 1 particle irreducible vacuum polarization diagrams. In perturbation theory $\Delta \alpha(s)$ is given by:

$$
\Delta \alpha(s)=\frac{\alpha}{3 \pi} \sum_{f} Q_{f}^{2} N_{C f}\left(\log \frac{2}{m_{f}^{2}}-\frac{5}{3}\right)
$$

where $N_{C f}=3$ for quarks and 1 for leptons. However, the perturbative formula is only reliable for leptons, not for quarks (because of the unknown values of the effective quark masses). Separating the leptonic, the light quark and the top quark contributions to $\Delta \alpha(s)$ we have:

$$
\Delta \alpha(s)=\Delta \alpha(s)_{\ell}+\Delta \alpha(s)_{h}+\Delta \alpha(s)_{t}
$$

with:

$$
\begin{aligned}
\Delta \alpha(s)_{\ell} & =0.0331421 ; \\
\Delta \alpha(s)_{t} & =\frac{\alpha}{3 \pi} \frac{4}{15} \frac{m_{Z}^{2}}{m_{t}^{2}}=-0.000061
\end{aligned}
$$

Note that in QED there is decoupling so that the top quark contribution approaches zero in the large $m_{t}$ limit. For $\Delta \alpha(s)_{h}$ one can use eq. (3.2i) and the Cauchy theorem to obtain the representation:

$$
\Delta \alpha\left(m_{Z}^{2}\right)_{h}=-\frac{\alpha m_{Z}^{2}}{3 \pi} \operatorname{Re} \int_{4 m_{\pi}^{2}}^{\infty} \frac{d s}{s} \frac{R(s)}{s-m_{Z}^{2}-i \epsilon}
$$

where $R(s)$ is the familiar ratio of the hadronic to the pointlike $\ell^{+} \ell^{-}$cross-section from photon exchange in $e^{+} e^{-}$annihilation. At $s$ large one can use the perturbative expansion for $R(s)$ while at small $s$ one can use the actual data. In recent years there has been a lot of activity on this subject and a number of independent new estimates of $\alpha\left(m_{Z}\right)$ have appeared in the literature [5in]. A consensus has been established and the value used at present is

$$
\alpha\left(m_{Z}\right)^{-1}=128.90 \pm 0.09
$$

As I said, for the derivation of this result the QCD theoretical prediction is actually used for large values of $\mathrm{s}$ where the data do not exist. But the sensitivity of the dispersive integral to this region is strongly suppressed, so that no important 
model dependence is introduced. More recently some analyses have appeared where one studied by how much the error on $\alpha_{s}\left(m_{Z}\right)$ is reduced by using the QCD prediction down to $\sqrt{s}=m_{\tau}$, with the possible exception of the regions around the charm and beauty thresholds [i6]. These attempts were motivated by the apparent success of QCD predictions in $\tau$ decays, despite the low $\tau$ mass (note however that the relevant currents are $\mathrm{V}$-A in $\tau$ decay but $\mathrm{V}$ in the present case). One finds that the central value is not much changed while the error in eq.(3.7) is reduced from 0.09 down to something like 0.03-0.04, but, of course, at the price of more model dependence. For this reason, in the following, we shall use the more conservative value in eq.(3.7).

As for the strong coupling $\alpha_{s}\left(m_{Z}\right)$ the world average central value is by now quite stable. The error is going down because the dispersion among the different measurements is much smaller in the most recent set of data. The most important determinations of $\alpha_{s}\left(m_{Z}\right)$ are summarised in table $2[\overline{7}]$. For all entries, the main sources of error are the theoretical ambiguities which are larger than the experimental errors. The only exception is the measurement from the electroweak precision tests, but only if one assumes that the SM electroweak sector is correct. My personal views on the theoretical errors are reflected in the table 2 . The error on the final average is taken by all authors between \pm 0.003 and \pm 0.005 depending on how conservative one is. Thus in the following our reference value will be

$$
\alpha_{s}\left(m_{Z}\right)=0.119 \pm 0.004
$$

Finally a few words on the current status of the direct measurement of $m_{t}$. The present combined $\mathrm{CDF} / \mathrm{D} 0$ result is

$$
m_{t}=173.8 \pm 5.0 \mathrm{GeV}
$$

The error is so small by now that one is approaching a level where a more careful investigation of the effects of colour rearrangement on the determination of $m_{t}$ is needed. One wants to determine the top quark mass, defined as the invariant mass of its decay products (i.e. $\mathrm{b}+\mathrm{W}+$ gluons + $\gamma$ 's). However, due to the need of colour rear- rangement, the top quark and its decay products cannot be really isolated from the rest of the event. Some smearing of the mass distribution is induced by this colour crosstalk which involves the decay products of the top, those of the antitop and also the fragments of the incoming (anti)protons. A reliable quantitative computation of the smearing effect on the $m_{t}$ determination is difficult because of the importance of non perturbative effects. An induced error of the order of $1 \mathrm{GeV}$ on $m_{t}$ could reasonably be expected. Thus further progress on the $m_{t}$ determination demands tackling this problem in more depth.

In order to appreciate the relative importance of the different sources of theoretical errors for precision tests of the SM, we report in table 3 a comparison for the most relevant observables. What is important to stress is that the ambiguity from $m_{t}$, once by far the largest one, is by now smaller than the error from $m_{H}$. We also see from table 3 that the error from $\Delta \alpha\left(m_{Z}\right)$ is expecially important for $\sin ^{2} \theta_{\text {eff }}$ and, to a lesser extent, is also sizeable for $\Gamma_{Z}$ and $\epsilon_{3}$.

The most important recent advance in the theory of radiative corrections is the calculation of the $o\left(g^{4} m_{t}^{2} / m_{W}^{2}\right)$ terms in $\sin ^{2} \theta_{e f f}, m_{W}$ and, more recently in $\delta \rho$ [isin]. The result implies a small but visible correction to the predicted values but expecially a seizable decrease of the ambiguity from scheme dependence (a typical effect of truncation). These callculations are now implemented in the fitting codes used in the analysis of LEP data. The fitted value of the Higgs mass is lowered by about $30 \mathrm{GeV}$ due to this effect.

We now discuss fitting the data in the SM. As the mass of the top quark is now rather precisely known from CDF and D0 one must distinguish two different types of fits. In one type one wants to answer the question: is $m_{t}$ from radiative corrections in agreement with the direct measurement at the Tevatron? Similarly how does $m_{W}$ inferred from radiative corrections compare with the direct measurements at the Tevatron and LEP2? For answering these interesting but somewhat limited questions, one must clearly exclude the measurements of $m_{t}$ and $m_{W}$ from the input set of data. Fitting all other 


\begin{tabular}{|l|ll|}
\hline Measurements & \multicolumn{2}{|c|}{$\alpha_{s}\left(m_{Z}\right)$} \\
\hline$R_{\tau}$ & $0.122 \pm 0.006$ & $(\mathrm{Th})$ \\
Deep Inelastic Scattering & $0.116 \pm 0.005 \quad(\mathrm{Th})$ \\
$Y_{\text {decay }}$ & $0.112 \pm 0.010 \quad(\mathrm{Th})$ \\
Lattice QCD & $0.117 \pm 0.007 \quad(\mathrm{Th})$ \\
$R e^{+} e^{-}(\sqrt{s}<62 \mathrm{GeV})$ & $0.124 \pm 0.021 \quad(\mathrm{Exp})$ \\
Fragmentation functions in $e^{+} e^{-}$ & $0.124 \pm 0.012 \quad(\mathrm{Th})$ \\
Jets in $e^{+} e^{-}$at and below the $Z$ & $0.121 \pm 0.008 \quad(\mathrm{Th})$ \\
$Z$ line shape (Assuming SM) & $0.120 \pm 0.004$ & $(\mathrm{Exp})$ \\
\hline
\end{tabular}

Table 2: Errors from different sources: $\Delta_{\text {now }}^{e x p}$ is the present experimental error; $\Delta \alpha^{-1}$ is the impact of $\Delta \alpha^{-1}= \pm 0.09 ; \Delta_{t h}$ is the estimated theoretical error from higher orders; $\Delta m_{t}$ is from $\Delta m_{t}= \pm 6 \mathrm{GeV} ; \Delta m_{H}$ is from $\Delta m_{H}=60-1000 \mathrm{GeV} ; \Delta \alpha_{s}$ corresponds to $\Delta \alpha_{s}= \pm 0.003$. The epsilon parameters are defined in Section 4.1

\begin{tabular}{|l|l|l|l|l|l|l|}
\hline Parameter & $\Delta_{\text {now }}^{\text {exp }}$ & $\Delta \alpha^{-1}$ & $\Delta_{t h}$ & $\Delta m_{t}$ & $\Delta m_{H}$ & $\Delta \alpha_{s}$ \\
\hline$\Gamma_{Z}(\mathrm{MeV})$ & \pm 2.4 & \pm 0.7 & \pm 0.8 & \pm 1.4 & \pm 4.6 & \pm 1.7 \\
$\sigma_{h}(\mathrm{pb})$ & 58 & 1 & 4.3 & 3.3 & 4 & 17 \\
$R_{h} \cdot 10^{3}$ & 26 & 4.3 & 5 & 2 & 13.5 & 20 \\
$\Gamma_{l}(\mathrm{keV})$ & 100 & 11 & 15 & 55 & 120 & 3.5 \\
$A_{F B}^{l} \cdot 10^{4}$ & 9.6 & 4.2 & 1.3 & 3.3 & 13 & 0.18 \\
$\sin ^{2} \theta \cdot 10^{4}$ & 19 & 2.3 & 0.8 & 1.9 & 7.5 & 0.1 \\
$m_{W}(\mathrm{MeV})$ & 60 & 12 & 9 & 37 & 100 & 2.2 \\
$R_{b} \cdot 10^{4}$ & 7.4 & 0.1 & 1 & 2.1 & 0.25 & 0 \\
$\epsilon_{1} \cdot 10^{3}$ & 1.2 & & $\sim 0.1$ & & & 0.2 \\
$\epsilon_{3} \cdot 10^{3}$ & 1.2 & 0.5 & $\sim 0.1$ & & & 0.12 \\
$\epsilon_{b} \cdot 10^{3}$ & 2.1 & & $\sim 0.1$ & & & 1 \\
\hline
\end{tabular}

Table 3: Errors from different sources: $\Delta_{\text {now }}^{e x p}$ is the present experimental error; $\Delta \alpha^{-1}$ is the impact of $\Delta \alpha^{-1}= \pm 0.09 ; \Delta_{t h}$ is the estimated theoretical error from higher orders; $\Delta m_{t}$ is from $\Delta m_{t}= \pm 6 G e V ; \Delta m_{H}$ is from $\Delta m_{H}=60-1000 \mathrm{GeV} ; \Delta \alpha_{s}$ corresponds to $\Delta \alpha_{s}= \pm 0.003$. The epsilon parameters are defined in Section 4.1

data in terms of $m_{t}, m_{H}$ and $\alpha_{s}\left(m_{Z}\right)$ one finds the results shown in the second column of table 4 [3. 3 . The extracted value of $m_{t}$ is typically a bit too low. For example, as shown in the table 4 , from all the electroweak data except the direct production results on $m_{t}$ and $m_{W}$, one finds $m_{t}=158 \pm 9 \mathrm{GeV}$. There is a strong correlation between $m_{t}$ and $m_{H}$. $\sin ^{2} \theta_{\text {eff }}$ and $m_{W}$ drive the fit to small values of $m_{H}$. Then, at small $m_{H}$ the widths, in particular the leptonic width (whose prediction is nearly independent of $\alpha_{s}$ ) drive the fit to small $m_{t}$. In a more general type of fit, e.g. for determining the overall consistency of the SM or the best present estimate for some quantity, say $m_{W}$, one should of course not ig- nore the existing direct determinations of $m_{t}$ and $m_{W}$. Then, from all the available data, by fitting $m_{t}, m_{H}$ and $\alpha_{s}\left(m_{Z}\right)$ one finds the values shown in the last column of table 4 .

This is the fit also referred to in table 1 . The corresponding fitted values of $\sin ^{2} \theta_{\text {eff }}$ and $m_{W}$ are:

$$
\begin{aligned}
\sin ^{2} \theta_{e f f} & =0.23156 \pm 0.00019 \\
m_{W} & =80.370 \pm 0.027 \mathrm{GeV}
\end{aligned}
$$

The fitted value of $\sin ^{2} \theta_{\text {eff }}$ is practically identical to the LEP+SLD average. The error of 27 $\mathrm{MeV}$ on $m_{W}$ clearly sets up a goal for the direct measurement of $m_{W}$ at LEP2 and the Tevatron. 


\begin{tabular}{|l|l|l|l|}
\hline Parameter & LEP $\left(\right.$ incl. $\left.m_{W}\right)$ & All but $m_{W}, m_{t}$ & All Data \\
\hline$m_{t}(\mathrm{GeV})$ & $160+13-10$ & $158+9-8$ & $171.3 \pm 4.9$ \\
$m_{H}(\mathrm{GeV})$ & $66+142-38$ & $34+45-16$ & $84+91-51$ \\
$\log \left[m_{H}(\mathrm{GeV})\right]$ & $1.82+0.50-0.37$ & $1.53+0.37-0.28$ & $1.92+0.32-0.41$ \\
$\alpha_{s}\left(m_{Z}\right)$ & $0.121 \pm 0.003$ & $0.120 \pm 0.003$ & $0.119 \pm 0.003$ \\
$\chi^{2} /$ dof & $4.2 / 9$ & $14 / 12$ & $16.4 / 15$ \\
\hline
\end{tabular}

Table 4: Standard Model fits of electroweak data

As a final comment we want to recall that the radiative corrections are functions of $\log \left(m_{H}\right)$. It is truly remarkable that the fitted value of $\log \left(m_{H}\right)$ is found to fall right into the very narrow allowed window around the value 2 specified by the lower limit from direct searches, $m_{H}>\sim$ $90 \mathrm{GeV}$, and the theoretical upper limit in the SM $m_{H}<600-800 \mathrm{GeV}$ (see later). Note that if the Higgs is removed from the theory, $\log m_{H} \rightarrow \log \Lambda+$ constant, where $\Lambda$ is a cutoff or the scale of the new physics that replaces the Higgs. The control of the finite terms is lost. Thus the fact that from experiment, one finds $\log m_{H} \sim 2$ is a strong argument in favour of the precise form of the Higgs mechanism as in the SM. The fulfilment of this very stringent consistency check is a beautiful argument in favour of a fundamental Higgs (or one with a compositeness scale much above the weak scale).

\section{A More General Analysis of Elec- troweak Data}

We now discuss an update of the epsilon analysis [4].] which is a method to look at the data in a more general context than the SM. The starting point is to isolate from the data that part which is due to the purely weak radiative corrections. In fact the epsilon variables are defined in such a way that they are zero in the approximation when only effects from the SM at the tree level plus pure QED and pure QCD corrections are taken into account. This very simple version of improved Born approximation is a good first approximation according to the data and is independent of $m_{t}$ and $m_{H}$. In fact the whole $m_{t}$ and $m_{H}$ dependence arises from weak loop corrections and therefore is only contained in the epsilon variables. Thus the epsilons are extracted from the data without need of specifying $m_{t}$ and $m_{H}$. But their predicted value in the SM or in any extension of it depend on $m_{t}$ and $m_{H}$. This is to be compared with the competitor method based on the S, T, U variables. The latter cannot be obtained from the data without specifying $m_{t}$ and $m_{H}$ because they are defined as deviations from the complete SM prediction for specified $m_{t}$ and $m_{H}$. Of course there are very many variables that vanish if pure weak loop corrections are neglected, at least one for each relevant observable. Thus for a useful definition we choose a set of representative observables that are used to parametrize those hot spots of the radiative corrections where new physics effects are most likely to show up. These sensitive weak correction terms include vacuum polarization diagrams which being potentially quadratically divergent are likely to contain all possible non decoupling effects (like the quadratic top quark mass dependence in the SM). There are three independent vacuum polarization contributions. In the same spirit, one must add the $Z \rightarrow b \bar{b}$ vertex which also includes a large top mass dependence. Thus altogether we consider four defining observables: one asymmetry, for example $A_{F B}^{l}$, (as representative of the set of measurements that lead to the determination of $\sin ^{2} \theta_{\text {eff }}$ ), one width (the leptonic width $\Gamma_{l}$ is particularly suitable because it is practically independent of $\left.\alpha_{s}\right), m_{W}$ and $R_{b}$. Here lepton universality has been taken for granted, because the data show that it is verified within the present accuracy. The four variables, $\epsilon_{1}, \epsilon_{2}, \epsilon_{3}$ and $\epsilon_{b}$ are defined in one to one correspondence with the set of observables $A_{l}^{F B}$, $\Gamma_{l}, m_{W}$, and $R_{b}$. The definition is so chosen that the quadratic top mass dependence is only present in $\epsilon_{1}$ and $\epsilon_{b}$, while the $m_{t}$ dependence of 
$\epsilon_{2}$ and $\epsilon_{3}$ is logarithmic. The definition of $\epsilon_{1}$ and $\epsilon_{3}$ is specified in terms of $A_{l}^{F B}$ and $\Gamma_{l}$ only. Then adding $m_{W}$ or $R_{b}$ one obtains $\epsilon_{2}$ or $\epsilon_{b}$. We now specify the relevant definitions in detail.

\subsection{Basic Definitions and Results}

We start from the basic observables $m_{W} / m_{Z}, \Gamma_{l}$ and $A_{l}^{F B}$ and $\Gamma_{b}$. From these four quantities one can isolate the corresponding dynamically significant corrections $\Delta r_{W}, \Delta \rho, \Delta k$ and $\epsilon_{b}$, which contain the small effects one is trying to disentangle and are defined in the following. First we introduce $\Delta r_{W}$ as obtained from $m_{W} / m_{Z}$ by the relation:

$$
\left(1-\frac{m_{W}^{2}}{m_{Z}^{2}}\right) \frac{m_{W}^{2}}{m_{Z}^{2}}=\frac{\pi \alpha\left(m_{Z}\right)}{\sqrt{2} G_{F} m_{Z}^{2}\left(1-\Delta r_{W}\right)}
$$

Here $\alpha\left(m_{Z}\right)=\alpha /(1-\Delta \alpha)$ is fixed to the central value $1 / 128.90$ so that the effect of the running of $\alpha$ due to known physics is extracted from $1-$ $\Delta r=(1-\Delta \alpha)\left(1-\Delta r_{W}\right)$. In fact, the error on $1 / \alpha\left(m_{Z}\right)$, as given in eq.(3.7.) would then affect $\Delta r_{W}$. In order to define $\Delta \rho$ and $\Delta k$ we consider the effective vector and axial-vector couplings $g_{V}$ and $g_{A}$ of the on-shell $\mathrm{Z}$ to charged leptons, given by the formulae:

$$
\begin{aligned}
\Gamma_{l} & =\frac{G_{F} m_{Z}^{3}}{6 \pi \sqrt{2}}\left(g_{V}^{2}+g_{A}^{2}\right)\left(1+\frac{3 \alpha}{4 \pi}\right), \\
A_{l}^{F B}\left(\sqrt{s}=m_{Z}\right) & =\frac{3 g_{V}^{2} g_{A}^{2}}{\left(g_{V}^{2}+g_{A}^{2}\right)^{2}} \\
& =\frac{3 x^{2}}{\left(1+x^{2}\right)^{2}} .
\end{aligned}
$$

Note that $\Gamma_{l}$ stands for the inclusive partial width $\Gamma(Z \rightarrow l \bar{l}+$ photons $)$. We stress the following points. First, we have extracted from $\left(g_{V}^{2}+g_{A}^{2}\right)$ the factor $(1+3 \alpha / 4 \pi)$ which is induced in $\Gamma_{l}$ from final state radiation. Second, by the asymmetry at the peak in eq.(4.21) we mean the quantity which is commonly referred to by the LEP experiments (denoted as $A_{F B}^{0}$ in ref. [30 is corrected for all QED effects, including initial and final state radiation and also for the effect of the imaginary part of the $\gamma$ vacuum polarization diagram. In terms of $g_{A}$ and $x=g_{V} / g_{A}$, the quantities $\Delta \rho$ and $\Delta k$ are given by:

$g_{A}=-\frac{\sqrt{\rho}}{2} \sim-\frac{1}{2}\left(1+\frac{\Delta \rho}{2}\right)$,

$$
x=\frac{g_{V}}{g_{A}}=1-4 \sin ^{2} \theta_{e f f}=1-4(1+\Delta k) s_{0}^{2} .
$$

Here $s_{0}^{2}$ is $\sin ^{2} \theta_{\text {eff }}$ before non pure-QED corrections, given by:

$$
s_{0}^{2} c_{0}^{2}=\frac{\pi \alpha\left(m_{Z}\right)}{\sqrt{2} G_{F} m_{Z}^{2}}
$$

with $c_{0}^{2}=1-s_{0}^{2}\left(s_{0}^{2}=0.231095\right.$ for $\left.m_{Z}=91.188 \mathrm{GeV}\right)$.

We now define $\epsilon_{b}$ from $\Gamma_{b}$, the inclusive partial width for $Z \rightarrow b \bar{b}$ according to the relation

$$
\begin{aligned}
\Gamma_{b}= & \frac{G_{F} m_{Z}^{3}}{6 \pi \sqrt{2}} \beta\left(\frac{3-\beta^{2}}{2} g_{b V}^{2}+\beta^{2} g_{b A}^{2}\right) \\
& N_{C} R_{Q C D}\left(1+\frac{\alpha}{12 \pi}\right)
\end{aligned}
$$

where $N_{C}=3$ is the number of colours, $\beta=$ $\sqrt{1-4 m_{b}^{2} / m_{Z}^{2}}$, with $m_{b}=4.7 \mathrm{GeV}, R_{Q C D}$ is the QCD correction factor given by

$R_{Q C D}=1+1.2 a-1.1 a^{2}-13 a^{3} ; \quad a=\frac{\alpha_{s}\left(m_{Z}\right)}{\pi}$

and $g_{b V}$ and $g_{b A}$ are specified as follows

$$
\begin{aligned}
& g_{b A}=-\frac{1}{2}\left(1+\frac{\Delta \rho}{2}\right)\left(1+\epsilon_{b}\right), \\
& \frac{g_{b V}}{g_{b A}}=\frac{1-4 / 3 \sin ^{2} \theta_{e f f}+\epsilon_{b}}{1+\epsilon_{b}} .
\end{aligned}
$$

This is clearly not the most general deviation from the SM in the $Z \rightarrow b \bar{b}$ but $\epsilon_{b}$ is closely related to the quantity $-\operatorname{Re}\left(\delta_{b-v e r t e x}\right)$ where the large $m_{t}$ corrections are located in the SM.

As is well known, in the SM the quantities $\Delta r_{W}, \Delta \rho, \Delta k$ and $\epsilon_{b}$, for sufficiently large $m_{t}$, are all dominated by quadratic terms in $m_{t}$ of order $G_{F} m_{t}^{2}$. As new physics can more easily be disentangled if not masked by large conventional $m_{t}$ effects, it is convenient to keep $\Delta \rho$ and $\epsilon_{b}$ while trading $\Delta r_{W}$ and $\Delta k$ for two quantities with no contributions of order $G_{F} m_{t}^{2}$. We thus introduce the following linear combinations:

$$
\begin{aligned}
& \epsilon_{1}=\Delta \rho, \\
& \epsilon_{2}=c_{0}^{2} \Delta \rho+\frac{s_{0}^{2} \Delta r_{W}}{c_{0}^{2}-s_{0}^{2}}-2 s_{0}^{2} \Delta k, \\
& \epsilon_{3}=c_{0}^{2} \Delta \rho+\left(c_{0}^{2}-s_{0}^{2}\right) \Delta k .
\end{aligned}
$$

The quantities $\epsilon_{2}$ and $\epsilon_{3}$ no longer contain terms of order $G_{F} m_{t}^{2}$ but only logarithmic terms in $m_{t}$. 
The leading terms for large Higgs mass, which are logarithmic, are contained in $\epsilon_{1}$ and $\epsilon_{3}$. In the Standard Model one has the following "large" asymptotic contributions:

$$
\begin{aligned}
\epsilon_{1} & =\frac{3 G_{F} m_{t}^{2}}{8 \pi^{2} \sqrt{2}}-\frac{3 G_{F} m_{W}^{2}}{4 \pi^{2} \sqrt{2}} \tan ^{2} \theta_{W} \ln \frac{m_{H}}{m_{Z}}+\ldots, \\
\epsilon_{2} & =-\frac{G_{F} m_{W}^{2}}{2 \pi^{2} \sqrt{2}} \ln \frac{m_{t}}{m_{Z}}+\ldots, \\
\epsilon_{3} & =\frac{G_{F} m_{W}^{2}}{12 \pi^{2} \sqrt{2}} \ln \frac{m_{H}}{m_{Z}}-\frac{G_{F} m_{W}^{2}}{6 \pi^{2} \sqrt{2}} \ln \frac{m_{t}}{m_{Z}} \ldots, \\
\epsilon_{b} & =-\frac{G_{F} m_{t}^{2}}{4 \pi^{2} \sqrt{2}}+\ldots
\end{aligned}
$$

The relations between the basic observables and the epsilons can be linearised, leading to the approximate formulae

$$
\begin{aligned}
\frac{m_{W}^{2}}{m_{Z}^{2}} & =\left.\frac{m_{W}^{2}}{m_{Z}^{2}}\right|_{B}\left(1+1.43 \epsilon_{1}-1.00 \epsilon_{2}-0.86 \epsilon_{3}\right), \\
\Gamma_{l} & =\left.\Gamma_{l}\right|_{B}\left(1+1.20 \epsilon_{1}-0.26 \epsilon_{3}\right) \\
A_{l}^{F B} & =\left.A_{l}^{F B}\right|_{B}\left(1+34.72 \epsilon_{1}-45.15 \epsilon_{3}\right), \\
\Gamma_{b} & =\left.\Gamma_{b}\right|_{B}\left(1+1.42 \epsilon_{1}-0.54 \epsilon_{3}+2.29 \epsilon_{b}\right) .
\end{aligned}
$$

The Born approximations, as defined above, depend on $\alpha_{s}\left(m_{Z}\right)$ and also on $\alpha\left(m_{Z}\right)$. Defining

$\delta \alpha_{s}=\frac{\alpha_{s}\left(m_{Z}\right)-0.119}{\pi} ; \delta \alpha=\frac{\alpha\left(m_{Z}\right)-\frac{1}{128.90}}{\alpha}$

we have

$$
\begin{aligned}
\left.\frac{m_{W}^{2}}{m_{Z}^{2}}\right|_{B} & =0.768905(1-0.40 \delta \alpha) \\
\left.\Gamma_{l}\right|_{B} & =83.563(1-0.19 \delta \alpha) \mathrm{MeV} \\
\left.A_{l}^{F B}\right|_{B} & =0.01696(1-34 \delta \alpha) \\
\left.\Gamma_{b}\right|_{B} & =379.8\left(1+1.0 \delta \alpha_{s}-0.42 \delta \alpha\right) .
\end{aligned}
$$

Note that the dependence on $\delta \alpha_{s}$ for $\left.\Gamma_{b}\right|_{B}$, shown

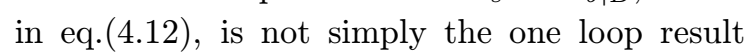
for $m_{b}=0$ but a combined effective shift which takes into account both finite mass effects and the contribution of the known higher order terms.

The important property of the epsilons is that, in the Standard Model, for all observables at the $\mathrm{Z}$ pole, the whole dependence on $m_{t}$ (and $m_{H}$ ) arising from one-loop diagrams only enters through the epsilons. The same is actually true, at the relevant level of precision, for all higher order $m_{t}$-dependent corrections. Actually, the only residual $m_{t}$ dependence of the various observables not included in the epsilons is in the terms of order $\alpha_{s}^{2}\left(m_{Z}\right)$ in the pure QCD correction factors to the hadronic widths. But this one is quantitatively irrelevant, especially in view of the errors connected to the uncertainty on the value of $\alpha_{s}\left(m_{Z}\right)$. The theoretical values of the epsilons in the SM from state of the art radiative corrections, also including the recent devel-

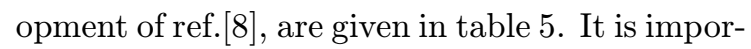
tant to remark that the theoretical values of the epsilons in the SM, as given in table 5, are not affected, at the percent level or so, by reasonable variations of $\alpha_{s}\left(m_{Z}\right)$ and/or $\alpha\left(m_{Z}\right)$ around their central values. By our definitions, in fact, no terms of order $\alpha_{s}^{n}\left(m_{Z}\right)$ or $\alpha \ln m_{Z} / m$ contribute to the epsilons. In terms of the epsilons, the following expressions hold, within the SM, for the various precision observables

$$
\begin{aligned}
\Gamma_{T} & =\Gamma_{T 0}\left(1+1.35 \epsilon_{1}-0.46 \epsilon_{3}+0.35 \epsilon_{b}\right), \\
R & =R_{0}\left(1+0.28 \epsilon_{1}-0.36 \epsilon_{3}+0.50 \epsilon_{b}\right), \\
\sigma_{h} & =\sigma_{h 0}\left(1-0.03 \epsilon_{1}+0.04 \epsilon_{3}-0.20 \epsilon_{b}\right), \\
x & =x_{0}\left(1+17.6 \epsilon_{1}-22.9 \epsilon_{3}\right), \\
R_{b} & =R_{b 0}\left(1-0.06 \epsilon_{1}+0.07 \epsilon_{3}+1.79 \epsilon_{b}(4) 13\right)
\end{aligned}
$$

where $\mathrm{x}=g_{V} / g_{A}$ as obtained from $A_{l}^{F B}$. The quantities in eqs. (A. $\overline{1} 0 \overline{4}-\overline{1}$ ) are clearly not independent and the redundant information is reported for convenience. By comparison with the computed radiative corrections we obtain

$$
\begin{aligned}
\Gamma_{T 0} & =2489.46\left(1+0.73 \delta \alpha_{s}-0.35 \delta \alpha\right) M e V \\
R_{0} & =20.8228\left(1+1.05 \delta \alpha_{s}-0.28 \delta \alpha\right) \\
\sigma_{h 0} & =41.420\left(1-0.41 \delta \alpha_{s}+0.03 \delta \alpha\right) n b \\
x_{0} & =0.075619-1.32 \delta \alpha \\
R_{b 0} & =0.2182355
\end{aligned}
$$

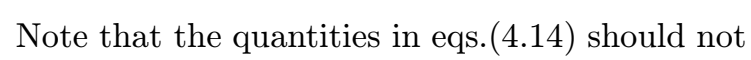
be confused, at least in principle, with the corresponding Born approximations, due to small "non universal" electroweak corrections. In practice, at the relevant level of approximation, the difference between the two corresponding quantities is in any case significantly smaller than the present experimental error. 


\begin{tabular}{|c|c|c|c|c|c|c|c|c|c|c|}
\hline$m_{t}$ & \multicolumn{3}{|c|}{$\epsilon_{1}$} & \multicolumn{3}{c|}{$\epsilon_{2}$} & \multicolumn{3}{c|}{$\epsilon_{3}$} & \multicolumn{3}{c|}{$\epsilon_{b}$} \\
$(\mathrm{GeV})$ & \multicolumn{2}{|c|}{$m_{H}(\mathrm{GeV})=$} & \multicolumn{3}{c|}{$m_{H}(\mathrm{GeV})=$} & \multicolumn{3}{c}{$m_{H}(\mathrm{GeV})=$} & All $m_{H}$ \\
& 70 & 300 & 1000 & 70 & 300 & 1000 & 70 & 300 & 1000 & \\
\hline 150 & 3.55 & 2.86 & 1.72 & -6.85 & -6.46 & -5.95 & 4.98 & 6.22 & 6.81 & -4.50 \\
160 & 4.37 & 3.66 & 2.50 & -7.12 & -6.72 & -6.20 & 4.96 & 6.18 & 6.75 & -5.31 \\
170 & 5.26 & 4.52 & 3.32 & -7.43 & -7.01 & -6.49 & 4.94 & 6.14 & 6.69 & -6.17 \\
180 & 6.19 & 5.42 & 4.18 & -7.77 & -7.35 & -6.82 & 4.91 & 6.09 & 6.61 & -7.08 \\
190 & 7.18 & 6.35 & 5.09 & -8.15 & -7.75 & -7.20 & 4.89 & 6.03 & 6.52 & -8.03 \\
200 & 8.22 & 7.34 & 6.04 & -8.59 & -8.18 & -7.63 & 4.87 & 5.97 & 6.43 & -9.01 \\
\hline
\end{tabular}

Table 5: Values of the epsilons in the SM as functions of $m_{t}$ and $m_{H}$ as obtained from recent versions of ZFITTER and TOPAZ0. These values (in $10^{-3}$ units) are obtained for $\alpha_{s}\left(m_{Z}\right)=0.119, \alpha\left(m_{Z}\right)=1 / 128.90$, but the theoretical predictions are essentially independent of $\alpha_{s}\left(m_{Z}\right)$ and $\alpha\left(m_{Z}\right)$

In principle, any four observables could have been picked up as defining variables. In practice we choose those that have a more clear physical significance and are more effective in the determination of the epsilons. In fact, since $\Gamma_{b}$ is actually measured by $R_{b}$ (which is nearly insensitive to $\alpha_{s}$ ), it is preferable to use directly $R_{b}$ itself as defining variable, as we shall do hereafter. In practice, since the value in eq.('1.14) is practically indistinguishable from the Born approximation of $R_{b}$, this determines no change in any of the equations given above but simply requires the corresponding replacement among the defining relations of the epsilons.

\subsection{Experimental Determination of the Ep- silon Variables}

The values of the epsilons as obtained, following the specifications in the previous sect.4.1, from the defining variables $m_{W}, \Gamma_{l}, A_{l}^{F B}$ and $R_{b}$ are shown in the first column of table 6 .

To proceed further and include other measured observables in the analysis we need to make some dynamical assumptions. The minimum amount of model dependence is introduced by including other purely leptonic quantities at the $\mathrm{Z}$ pole such as $A_{\tau}, A_{e}$ (measured from the angular dependence of the $\tau$ polarization) and $A_{L R}$ (measured by SLD). For this step, one is simply assuming that the different leptonic asymmetries are equivalent measurements of $\sin ^{2} \theta_{\text {eff }}$. We add, as usual, the measure of $A_{b}^{F B}$ because this observable is dominantly sensitive to the leptonic vertex. We then use the combined value of $\sin ^{2} \theta_{\text {eff }}$ obtained from the whole set of asymmetries measured at LEP and SLC given in eq.(2.1). At this stage the best values of the epsilons are shown in the second column of table 6 . In Figs. 2 port the $1 \sigma$ ellipses in the indicated $\epsilon_{i}-\epsilon_{j}$ planes that correspond to this set of input data.

All observables measured on the $\mathrm{Z}$ peak at LEP can be included in the analysis provided that we assume that all deviations from the SM are only contained in vacuum polarization diagrams (without demanding a truncation of the $q^{2}$ dependence of the corresponding functions) and/or the $Z \rightarrow b \bar{b}$ vertex. From a global fit of the data on $m_{W}, \Gamma_{T}, R_{h}, \sigma_{h}, R_{b}$ and $\sin ^{2} \theta_{\text {eff }}$ (for LEP data, we have taken the correlation matrix for $\Gamma_{T}, R_{h}$ and $\sigma_{h}$ given by the LEP experiments [3]., while we have considered the additional information on $R_{b}$ and $\sin ^{2} \theta_{\text {eff }}$ as independent) we obtain the values shown in the third column of table 6 . The comparison of theory and experiment at this stage is also shown in Figs. $\overline{2}_{-}^{-}$ $\bar{p}_{\mathbf{r}}^{\prime}$ More detailed information is shown in Fig. "6i", which refers to the level when also hadronic data are taken into account. But in fig.9 we compare the results obtained if $\sin ^{2} \theta_{\text {eff }}$ is extracted in turn from different asymmetries among those listed in Fig. $\overline{1}$. The ellipse marked "average" is the same as the one labelled "All high en." in Fig. $\bar{\beta}_{1}^{1}$ and corresponds to the value of $\sin ^{2} \theta_{\text {eff }}$ which is shown on the figure (and in eq.(2.2. We confirm that the value from $A_{L R}$ is far away 


\begin{tabular}{|ll|l|l|l|l|}
\hline$\epsilon$ & $10^{3}$ & Only def. quantities & All asymmetries & All High Energy & All Data \\
\hline$\epsilon_{1}$ & $10^{3}$ & $4.1 \pm 1.2$ & $4.3 \pm 1.2$ & $4.0 \pm 1.1$ & $3.7 \pm 1.1$ \\
$\epsilon_{2}$ & $10^{3}$ & $-8.2 \pm 2.1$ & $-8.8 \pm 1.9$ & $-9.0 \pm 2.0$ & $-9.3 \pm 2.0$ \\
$\epsilon_{3}$ & $10^{3}$ & $3.3 \pm 1.9$ & $4.4 \pm 1.2$ & $4.2 \pm 1.1$ & $3.9 \pm 1.1$ \\
$\epsilon_{b}$ & $10^{3}$ & $-4.3 \pm 1.9$ & $-4.4 \pm 1.9$ & $-4.8 \pm 1.9$ & $-4.6 \pm 1.9$ \\
\hline
\end{tabular}

Table 6: Experimental values of the epsilons in the SM from different sets of data. These values (in $10^{-3}$ units) are obtained for $\alpha_{s}\left(m_{Z}\right)=0.119 \pm 0.003, \alpha\left(m_{Z}\right)=1 / 128.90 \pm 0.09$, the corresponding uncertainties being included in the quoted errors

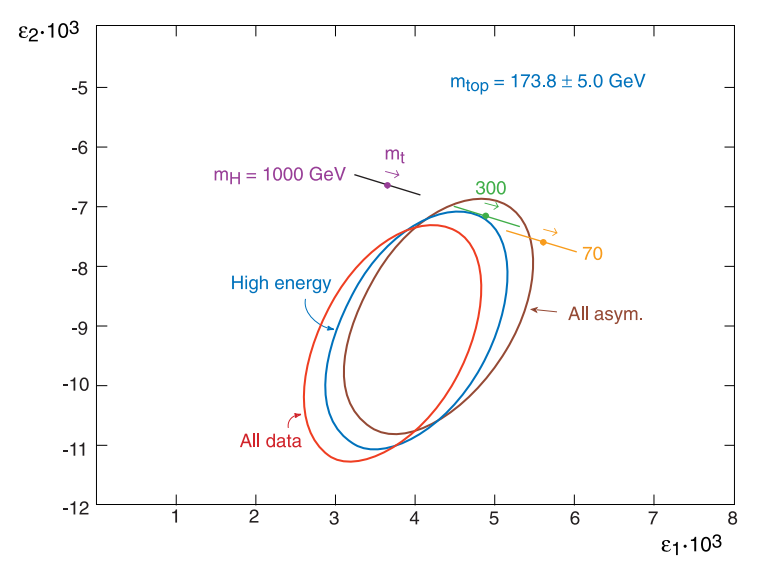

Figure 2: Data vs theory in the $\epsilon_{2}-\epsilon_{1}$ plane. The origin point corresponds to the "Born" approximation obtained from the SM at tree level plus pure QED and pure QCD corrections. The predictions of the full SM (also including the improvements of ref. $\overline{[}[\mathrm{i}]$ ) are shown for $m_{H}=70$, 300 and $1000 \mathrm{GeV}$ and $m_{t}=175.6 \pm 5.5 \mathrm{GeV}$ (a segment for each $m_{H}$ with the arrow showing the direction of $m_{t}$ increasing from $-1 \sigma$ to $+1 \sigma)$. The three $1-\sigma$ ellipses (38\% probability contours) are obtained from a) "All Asymm." $: \Gamma_{l}, m_{W}$ and $\sin ^{2} \theta_{\text {eff }}$ as obtained from the combined asymmetries (the value in eq. (2.10); b) "All High En.": the same as in a) plus all the hadronic variables at the Z; c) "All Data": the same as in b) plus the low energy data.

from the SM given the experimental value of $m_{t}$ and the bounds on $m_{H}$ and would correspond to very small values of $\epsilon_{3}$ and of $\epsilon_{1}$. We see also that while the $\tau$ FB asymmetry is also on the low side, the combined e and $\mu \mathrm{FB}$ asymmetry are right on top of the average. Finally the b FB asymmetry is on the high side.

To include in our analysis lower energy observables as well, a stronger hypothesis needs to be made: vacuum polarization diagrams are al-

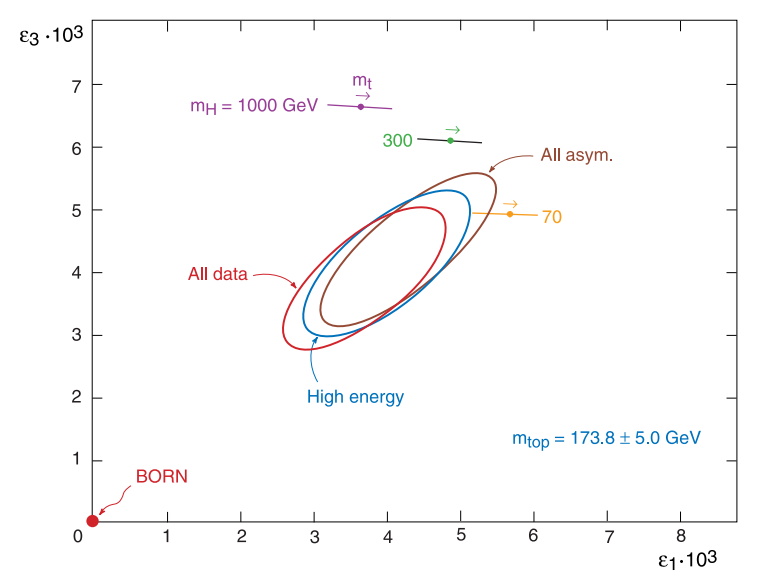

Figure 3: Data vs theory in the $\epsilon_{3}-\epsilon_{1}$ plane (notations as in Fig. 泪)

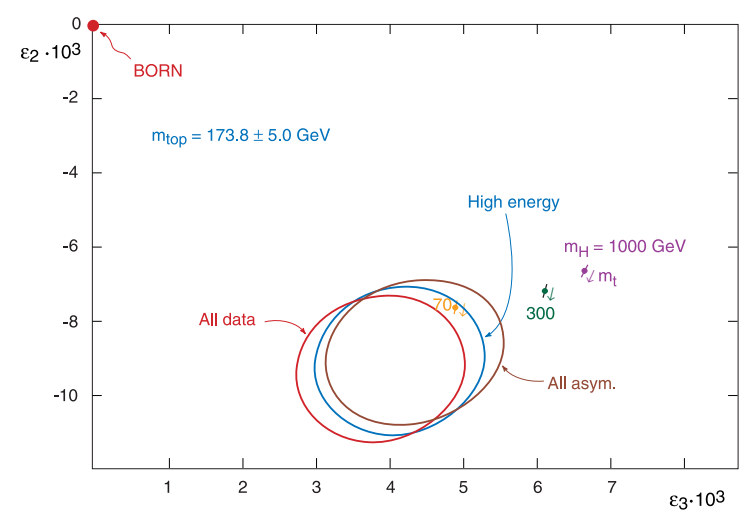

Figure 4: Data vs theory in the $\epsilon_{2}-\epsilon_{3}$ plane (notations as in Fig. 造)

lowed to vary from the SM only in their constant and first derivative terms in a $q^{2}$ expansion. In such a case, one can, for example, add to the analysis the ratio $R_{\nu}$ of neutral to charged current processes in deep inelastic neutrino scattering on nuclei [ $\left[\bar{p}_{1}^{\prime}\right],\left[10_{1}^{\prime}\right]$ the "weak charge" $Q_{W}$ measured in atomic parity violation experiments 


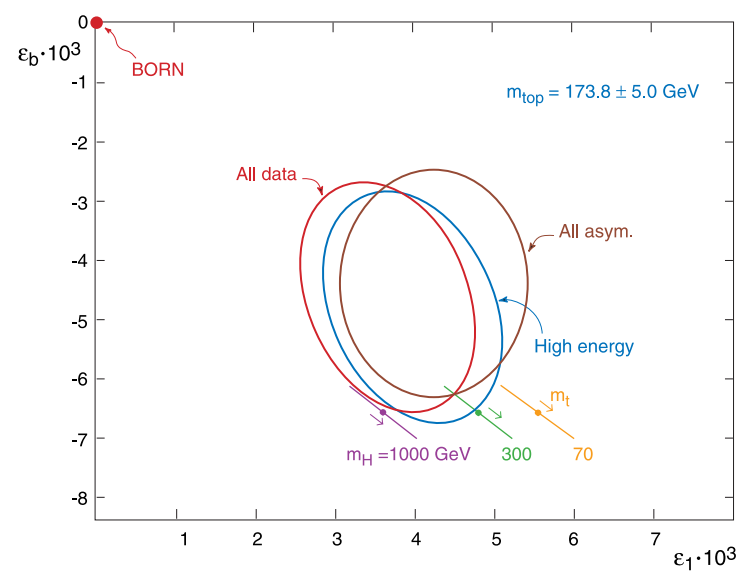

Figure 5: Data vs theory in the $\epsilon_{b}-\epsilon_{1}$ plane (notations as in Fig. 息)

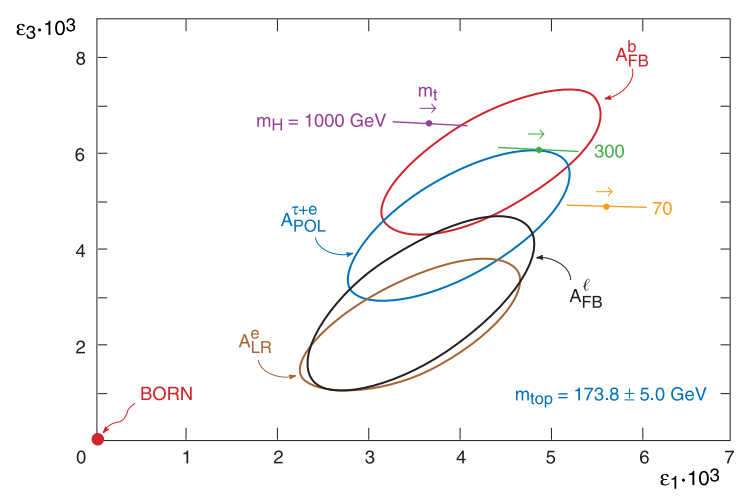

Figure 6: Data vs theory in the $\epsilon_{3}-\epsilon_{1}$ plane (notations as in Fig. $\left.{ }_{2}^{\prime}\right)$. The ellipse indicated with "Average" corresponds to the case "All high en" of Fig. $\overline{3}$ and is obtained from the combined value of $\sin ^{2} \theta_{\text {eff }}$. The other ellipses are obtained by replacing the combined $\sin ^{2} \theta_{\text {eff }}$ with the values obtained in turn from each individual asymmetry as shown by the labels.

on Cs [1] ind and the measurement of $g_{V} / g_{A}$ from $\nu_{\mu} e$ scattering [12in]. In this way one obtains the global fit given in the fourth column of table 6 and shown in Figs. $\overline{2}_{2}^{1}+\overline{5}_{i}^{1}$ In Fig. $\overline{7}_{1}$, we see the ellipse in the $\epsilon_{1}-\epsilon_{3}$ plane that is obtained from the low energy data by themselves. It is interesting that the tendency towards low values of $\epsilon_{1}$ and $\epsilon_{3}$ is present in the low energy data as in the high energy ones. Note that the low energy data by themselves are actually compatible with the "Born" approximation. With the progress of LEP the low energy data, while important as a check that no deviations from the expected $q^{2}$ dependence arise, play a lesser role in the global

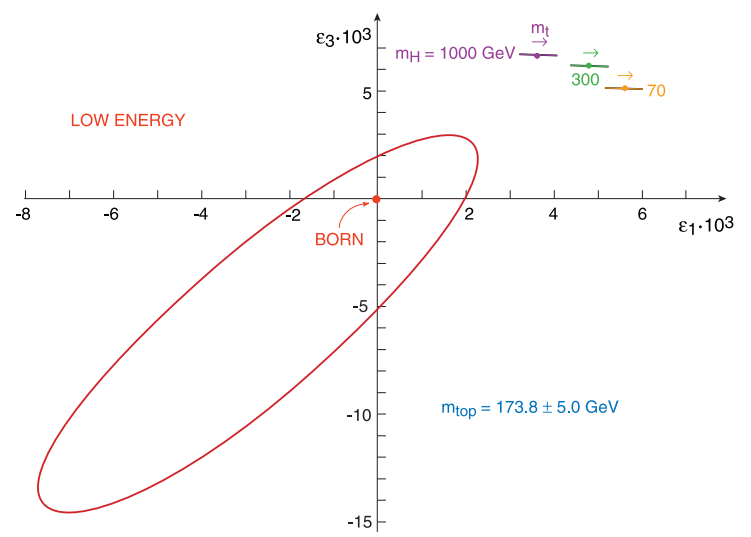

Figure 7: Data vs theory in the $\epsilon_{3}-\epsilon_{1}$ plane (notations as in Fig. $\left.{ }_{2}^{\prime}\right)$. Here the ellipse from the low energy data by themselves is plotted (deep inelastic neutrino scattering, atomic parity violation and $\nu_{\mu}-e$ scattering.

fit. This does not mean that they are not important. For example, the measured parity violation in atomic physics provides the best limits on possible new physics in the electron-quark sector. When HERA suggested the presence of leptoquarks, the limits from atomic parity violation practically excluded all possible parity violating four fermiom electron-quark contact terms. So low energy data are no more powerful enough to improve the determination of the parameters if the SM is assumed, but they are a very powerful constraint on new physics models. The best values of the $\epsilon$ 's from all the data are at present:

$$
\begin{aligned}
& \epsilon_{1} 10^{3}=3.7 \pm 1.1 \\
& \epsilon_{2} 10^{3}=-9.3 \pm 2.0 \\
& \epsilon_{3} 10^{3}=3.9 \pm 1.1 \\
& \epsilon_{b} 10^{3}=-4.6 \pm 1.9 .
\end{aligned}
$$

Note that the present ambiguity on the value of $\delta \alpha^{-1}\left(m_{Z}\right)= \pm 0.09$ corresponds to an uncertainty on $\epsilon_{3}$ (the other epsilons are not much affected) given by $\Delta \epsilon_{3} \quad 10^{3}= \pm 0.6$. Thus the theoretical error is still confortably less than the experimental error. In Fig. 4.2 we present a summary of the experimental values of the epsilons as compared to the SM predictions as functions of $m_{t}$ and $m_{H}$, which shows agreement within $1 \sigma$, but the central value of $\epsilon_{1}, \epsilon_{2}$ and $\epsilon_{3}$ are all low, 


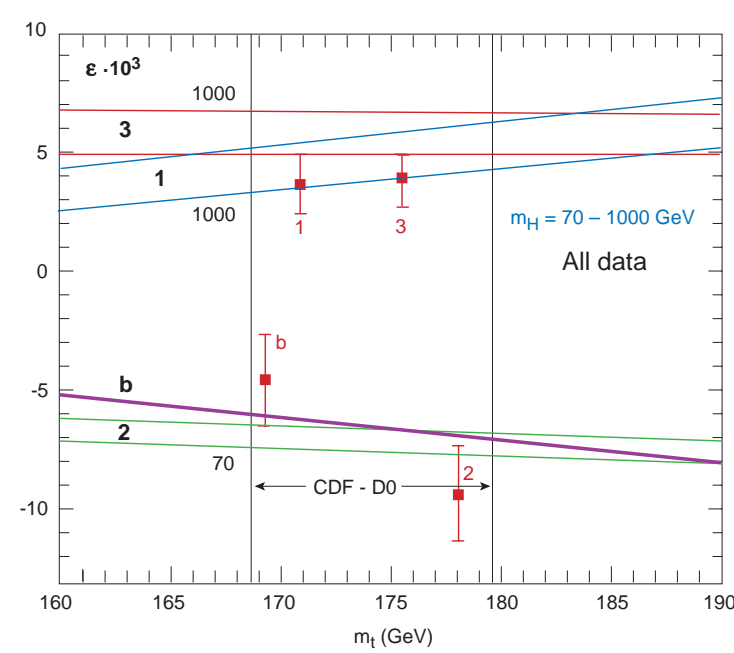

Figure 8: The bands (labeled by the $\epsilon$ index) are the predicted values of the epsilons in the $S M$ as functions of $m_{t}$ for $m_{H}=70-1000 \mathrm{GeV}$ (the $m_{H}$ value corresponding to one edge of the band is indicated). The CDF/D0 experimental 1- $\sigma$ range of $m_{t}$ is shown. The esperimental results for the epsilons from all data are displayed (from the last column of table 6). The position of the data on the $m_{t}$ axis has been arbitrarily chosen and has no particular meaning.

while the central value of $\epsilon_{b}$ is shifted upward with respect to the $\mathrm{SM}$ as a consequence of the still imperfect matching of $R_{b}$. A number of interesting features are clearly visible from Figs. $\overline{2}-$ 4.2. First, the good agreement with the SM and the evidence for weak corrections, measured by the distance of the data from the improved Born approximation point (based on tree level SM plus pure QED or QCD corrections). There is by now a solid evidence for departures from the improved Born approximation where all the epsilons vanish. In other words a clear evidence for the pure weak radiative corrections has been obtained and LEP/SLC are now measuring the various components of these radiative corrections. For example, some authors [1 $[13]$ have studied the sensitivity of the data to a particularly interesting subset of the weak radiative corrections, i.e. the purely bosonic part. These terms arise from virtual exchange of gauge bosons and Higgses. The result is that indeed the measurements are sufficiently precise to require the presence of these contributions in order to fit the data. Second, the gen- eral results of the SM fits are reobtained from a different perspective. We see the preference for light Higgs manifested by the tendency for $\epsilon_{3}$ to be rather on the low side. Since $\epsilon_{3}$ is practically independent of $m_{t}$, its low value demands $m_{H}$ small. If the Higgs is light then the preferred value of $m_{t}$ is somewhat lower than the Tevatron result (which in the epsilon analysis is not included among the input data). This is because also the value of $\epsilon_{1} \equiv \delta \rho$, which is determined by the widths, in particular by the leptonic width, is somewhat low. In fact $\epsilon_{1}$ increases with $m_{t}$ and, at fixed $m_{t}$, decreases with $m_{H}$, so that for small $m_{H}$ the low central value of $\epsilon_{1}$ pushes $m_{t}$ down. Note that also the central value of $\epsilon_{2}$ is on the low side, because the experimental value of $m_{W}$ is a little bit too large. Finally, we see that adding the hadronic quantities or the low energy observables hardly makes a difference in the $\epsilon_{i}-\epsilon_{j}$ plots with respect to the case with only the leptonic variables being included (the ellipse denoted by "All Asymm."). But, for example for the $\epsilon_{1}-\epsilon_{3}$ plot, while the leptonic ellipse contains the same information as one could obtain from a $\sin ^{2} \theta_{\text {eff }}$ vs $\Gamma_{l}$ plot, the content of the other two ellipses is much larger because it shows that the hadronic as well as the low energy quantities match the leptonic variables without need of any new physics. Note that the experimental values of $\epsilon_{1}$ and $\epsilon_{3}$ when the hadronic quantities are included also depend on the input value of $\alpha_{s}$ given in eq. $(\underline{3}-\overline{8} \cdot \overline{1})$.

The good agreement of the fitted epsilon values with the SM impose strong constraints on possible forms of new physics. Consider, for example, new quarks or leptons. Mass splitted multiplets contribute to $\Delta \epsilon_{1}$, in analogy to the t-b quark doublet. Recall that $\Delta \epsilon_{1} \sim+9.510^{-3}$ for the t-b doublet, which is about eight $\sigma$ 's in terms of the present error [1] 4]. Even mass degenerate multiplets are strongly constrained. They contribute to $\Delta \epsilon_{3}$ according to [i1

$$
\Delta \epsilon_{3} \sim N_{C} \frac{G_{F} m_{W}^{2}}{8 \pi^{2} \sqrt{2}} \frac{4}{3}\left(T_{3 L}-T_{3 R}\right)^{2}
$$

For example a new left-handed quark doublet, degenerate in mass, would contribute $\Delta \epsilon_{3} \sim+1.310^{-3}$, that is about one $\sigma$, but in the wrong direction, 
in the sense that the experimental value of $\epsilon_{3}$ favours a displacement, if any, with negative sign. Only vector fermions $\left(T_{3 L}=T_{3 R}\right)$ are not constrained. In particular, naive technicolour models , that introduce several new technifermions, are strongly disfavoured because they tend to produce large corrections with the wrong sign to $\epsilon_{1}, \epsilon_{3}$ and also to $\epsilon_{b}[\overline{1}$.

\section{The CKM Matrix}

Weak charged currents are the only tree level interactions in the SM that change flavour: by emission of a $\mathrm{W}$ an up-type quark is turned into a down-type quark, or a $\nu_{l}$ neutrino is turned into a $l^{-}$charged lepton (all fermions are lefthanded). If we start from an up quark that is a mass eigenstate, emission of a $\mathrm{W}$ turns it into a down-type quark state d' (the weak isospin partner of $u$ ) that in general is not a mass eigenstate. In general, the mass eigenstates and the weak eigenstates do not coincide and a unitary transformation connects the two sets:

$$
\left(\begin{array}{l}
d^{\prime} \\
s^{\prime} \\
b^{\prime}
\end{array}\right)=V\left(\begin{array}{l}
d \\
s \\
b
\end{array}\right)
$$

$\mathrm{V}$ is the Cabibbo-Kobayashi-Maskawa matrix. Thus in terms of mass eigenstates the charged weak current of quarks is of the form:

$$
J_{\mu}^{+} \propto \bar{u} \gamma_{\mu}\left(1-\gamma_{5}\right) t^{+} V d
$$

Since $\mathrm{V}$ is unitary (i.e. $V V^{\dagger}=V^{\dagger} V=1$ ) and commutes with $T^{2}, T_{3}$ and $\mathrm{Q}$ (because all d-type quarks have the same isospin and charge) the neutral current couplings are diagonal both in the primed and unprimed basis (if the $\mathrm{Z}$ downtype quark current is abbreviated as $\bar{d}^{\prime} \Gamma d^{\prime}$ then by changing basis we get $\bar{d} V^{\dagger} \Gamma V d$ and $\mathrm{V}$ and $\Gamma$ commute because $\Gamma$ is made of Dirac matrices and $T_{3}$ and $\mathrm{Q}$ generator matrices). This is the GIM mechanism that ensures natural flavour conservation of the neutral current couplings at the tree level.

For $\mathrm{N}$ generations of quarks, $\mathrm{V}$ is a $\mathrm{NxN}$ unitary matrix that depends on $N^{2}$ real numbers $\left(N^{2}\right.$ complex entries with $N^{2}$ unitarity constraints). However, the $2 N$ phases of up- and down-type quarks are not observable. Note that an overall phase drops away from the expression of the current in eq. (5.2), so that only $2 N-1$ phases can affect $\mathrm{V}$. In total, $\mathrm{V}$ depends on $N^{2}-$ $2 N+1=(N-1)^{2}$ real physical parameters. A similar counting gives $N(N-1) / 2$ as the number of independent parameters in an orthogonal $\mathrm{NxN}$ matrix. This implies that in $\mathrm{V}$ we have $N(N-$ 1)/2 mixing angles and $(N-1)^{2}-N(N-1) / 2$ phases: for $N=2$ one mixing angle (the Cabibbo angle) and no phase, for $N=3$ three angles and one phase etc.

Given the experimental near diagonal structure of $\mathrm{V}$ a convenient parametrisation is the one proposed by Maiani. One starts from the definition:

$$
\left|d^{\prime}\right\rangle=c_{13}\left|d_{C}\right\rangle+s_{13} e^{-i \phi}|b\rangle
$$

where $c_{13} \equiv \cos \theta_{13}, s_{13} \equiv \sin \theta_{13}$ (analogous shorthand notations will be used in the following), $d_{C}$ is the Cabibbo down quark and $\theta_{12} \equiv \theta_{C}$ is the Cabibbo angle (experimentally $s_{12} \equiv \lambda \sim$ $0.22)$.

$$
\left|d_{C}\right\rangle=c_{12}|d\rangle+s_{12}|s\rangle
$$

Note that in a four quark model the Cabibbo angle fixes both the ratio of couplings $(u \rightarrow d) /\left(\nu_{e} \rightarrow\right.$ $e)$ and the ratio of $(u \rightarrow d) /(u \rightarrow s)$. In a six quark model one has to choose which to keep as a definition of the Cabibbo angle. Here the second definition is taken and, in fact the $u \rightarrow d$ coupling is given by $V_{u d}=c_{13} c_{12}$ so that it is no longer specified by $\theta_{12}$ only. Also note that we can certainly fix the phases of $u, d, s$ so that a real coefficient appears in front of $d_{C}$ in eq. (15.3i). We now choose a basis of two orthonormal vectors, both orthogonal to $\left|d^{\prime}\right\rangle$ :

$$
\begin{aligned}
\left|s_{C}\right\rangle & =-s_{12}|d\rangle+c_{12}|s\rangle, \\
|v\rangle & =-s_{13} e^{i \phi}\left|d_{C}\right\rangle+c_{13}|b\rangle
\end{aligned}
$$

Here $\left|s_{C}\right\rangle$ is the Cabibbo s quark. Clearly s' and b' must be othonormal superpositions of the above base vectors defined in terms of an angle $\theta_{23}$ :

$\left|s^{\prime}\right\rangle=c_{23}\left|s_{C}\right\rangle+s_{23}|v\rangle, \quad\left|b^{\prime}\right\rangle=-s_{23}\left|s_{C}\right\rangle+c_{23}|v\rangle$

The general expression of $V_{i j}$ can be obtained from the above equations. But a considerable 
notational simplification is gained if one takes into account that from experiment we know that $s_{12} \equiv \lambda, s_{23} \sim o\left(\lambda^{2}\right)$ and $s_{13} \sim o\left(\lambda^{3}\right)$ are increasingly small and of the indicated orders of magnitude. Thus, following Wolfenstein one can set:

$s_{12} \equiv \lambda, \quad s_{23}=A \lambda^{2}, \quad s_{13} e^{-i \phi}=A \lambda^{3}(\rho-i \eta)$

As a result, by neglecting terms of higher order in $\lambda$ one can write down:

$$
\begin{aligned}
& V=\left[\begin{array}{ccc}
V_{u d} & V_{u s} & V_{u b} \\
V_{c d} & V_{c s} & V_{c b} \\
V_{t d} & V_{t s} & V_{t b}
\end{array}\right] \sim \\
& \sim\left[\begin{array}{ccc}
1-\frac{\lambda^{2}}{2} & \lambda & A \lambda^{3}(\rho-i \eta) \\
-\lambda & 1-\frac{\lambda^{2}}{2} & A \lambda^{2} \\
A \lambda^{3}(1-\rho-i \eta) & -A \lambda^{2} & 1
\end{array}\right] .
\end{aligned}
$$

Indicative values of the CKM parameters as obtained from experiment are (a survey of the current status of the CKM parameters can be found

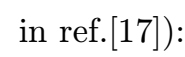

$$
\begin{array}{r}
\lambda=0.2196 \pm 0.0023 \\
A=0.82 \pm 0.04 \\
\sqrt{\rho^{2}+\eta^{2}}=0.4 \pm 0.1 ; \quad \eta \sim 0.3 \pm 0.2
\end{array}
$$

In the SM the non vanishing of the $\eta$ parameter is the only source of $\mathrm{CP}$ violation. The most direct and solid evidence for $\eta$ non vanishing is obtained from the measurement of $\epsilon$ in $\mathrm{K}$ decay. Unitarity of the CKM matrix $\mathrm{V}$ implies relations of the form $\sum_{a} V_{b a} V_{c a}^{*}=\delta_{b c}$. In most cases these relations do not imply particularly instructive constraints on the Wolfenstein parameters. But when the three terms in the sum are of comparable magnitude we get interesting information. The three numbers which must add to zero form a closed triangle in the complex plane, with sides of comparable length. This is the case for the $\mathrm{t}-\mathrm{u}$ triangle (Bjorken triangle) shown in Fig.

$$
V_{t d} V_{u d}^{*}+V_{t s} V_{u s}^{*}+V_{t b} V_{u b}^{*}=0
$$

All terms are of order $\lambda^{3}$. For $\eta=0$ the triangle would flatten down to vanishing area. In fact the area of the triangle, $\mathrm{J}$ of order $J \sim \eta A^{2} \lambda^{6}$, is

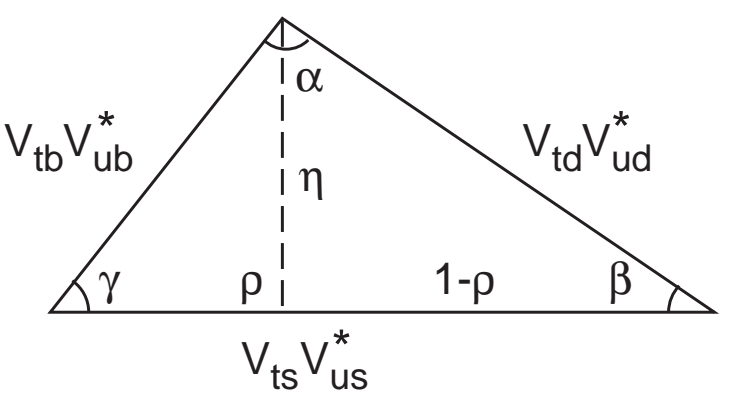

Figure 9: The Bjorken triangle corresponding to eq. $(\overline{5} \cdot \overline{1} \cdot \overline{1})$

the Jarlskog invariant (its value is independent of the parametrization). In the SM all CP violating observables must be proportional to J.

The physics of flavour will be explored in depth in the next few years at $\mathrm{DA} \phi \mathrm{NE}$ and at $B$-factories.

We have only discussed flavour mixing for quarks. But, clearly, if neutrino masses exist, as indicated by neutrino oscillations, then a similar mixing matrix must also be introduced in the leptonic sector (see section 6.2).

\section{Why Beyond the Standard Model?}

Given the striking success of the SM why are we not satisfied with that theory? Why not just find the Higgs particle, for completeness, and declare that particle physics is closed? The main reason is that there are strong conceptual indications for physics beyond the SM. There are also some phenomenological hints.

\subsection{Conceptual Problems with the Stan- dard Model}

It is considered highly unplausible that the origin of the electro-weak symmetry breaking can be explained by the standard Higgs mechanism, without accompanying new phenomena. New physics should be manifest at energies in the $\mathrm{TeV}$ domain. This conclusion follows fron an extrapolation of the SM at very high energies. The computed behaviour of the $S U(3) \otimes S U(2) \otimes U(1)$ 
couplings with energy clearly points towards the unification of the electro-weak and strong forces (Grand Unified Theories: GUTs) at scales of energy $M_{G U T} \sim 10^{14}-10^{16} \mathrm{GeV}$ which are close to the scale of quantum gravity, $M_{P l} \sim 10^{19} \mathrm{GeV}$ [1] 1 i]. One can also imagine a unified theory of all interactions also including gravity (at present superstrings provide the best attempt at such a theory). Thus GUTs and the realm of quantum gravity set a very distant energy horizon that modern particle theory cannot anymore ignore. Can the SM without new physics be valid up to such large energies? This appears unlikely because the structure of the SM could not naturally explain the relative smallness of the weak scale of mass, set by the Higgs mechanism at $m \sim 1 / \sqrt{G_{F}} \sim 250 \mathrm{GeV}$ with $G_{F}$ being the Fermi coupling constant. The weak scale $\mathrm{m}$ is $\sim 10^{17}$ times smaller than $M_{P l}$. Even if the weak scale is set near $250 \mathrm{GeV}$ at the classical level, quantum fluctuations would naturally shift it up to where new physics starts to apply, in particular up to $M_{P l}$ if there was no new physics up to gravity. This so-called hierarchy problem [1 $\left.{ }_{1}^{1}{ }_{1}^{\prime}\right]$ is related to the presence of fundamental scalar fields in the theory with quadratic mass divergences and no protective extra symmetry at $\mathrm{m}=0$. For fermions, first, the divergences are logaritmic and, second, at $\mathrm{m}=0$ an additional symmetry, i.e. chiral symmetry, is restored. Here, when talking of divergences we are not worried of actual infinities. The theory is renormalisable and finite once the dependence on the cut off is absorbed in a redefinition of masses and couplings. Rather the hierarchy problem is one of naturalness. If we consider the cut off as a manifestation of new physics that will modify the theory at large energy scales, then it is relevant to look at the dependence of physical quantities on the cut off and to demand that no unexplained enormously accurate cancellation arise.

According to the above argument the observed value of $m \sim 250 \mathrm{GeV}$ is indicative of the existence of new physics nearby. There are two main possibilities. Either there exist fundamental scalar Higgses but the theory is stabilised by supersymmetry, the boson-fermion symmetry that would downgrade the degree of divergence from quadratic to logarithmic. For approximate supersymmetry the cut off is replaced by the splitting between the normal particles and their supersymmetric partners. Then naturalness demands that this splitting (times the size of the weak gauge coupling) is of the order of the weak scale of mass, i.e. the separation within supermultiplets should be of the order of no more than a few $\mathrm{TeV}$. In this case the masses of most supersymmetric partners of the known particles, a very large managerie of states, would fall, at least in part, in the discovery reach of the LHC. There are consistent, fully formulated field theories constructed on the basis of this idea, the simplest one being the MSSM [20 ]. Note that all normal observed states are those whose masses are forbidden in the limit of exact $S U(2) \otimes U(1)$. Instead for all SUSY partners the masses are allowed in that limit. Thus when supersymmetry is broken in the $\mathrm{TeV}$ range but $S U(2) \otimes U(1)$ is intact only s-partners take mass while all normal particles remain massless. Only at the lower weak scale the masses of ordinary particles are generated. Thus a simple criterium exists to understand the difference between particles and s-particles.

The other main avenue is compositeness of some sort. The Higgs boson is not elementary but either a bound state of fermions or a condensate, due to a new strong force, much stronger than the usual strong interactions, responsible for the attraction. A plethora of new "hadrons", bound by the new strong force would exist in the LHC range. A serious problem for this idea is that nobody sofar has been able to build up a realistic model along these lines, but that could eventually be explained by a lack of ingenuity on the theorists side. The most appealing examples are technicolor theories [1] 1 in. These models where inspired by the breaking of chiral symmetry in massless QCD induced by quark condensates. In the case of the electroweak breaking new heavy techniquarks must be introduced and the scale analogous to $\Lambda_{Q C D}$ must be about three orders of magnitude larger. The presence of such a large force relatively nearby has a strong tendency to clash with the results of the electroweak precision tests. Another interesting idea is to replace the Higgs by a $t \bar{t}$ condensate [2 $\overline{1} \overline{1}]$. The Yukawa 
coupling of the Higgs to the $t \bar{t}$ pair becomes a four fermion $\bar{t} t \bar{t} t$ coupling with the corresponding strenght. The strong force is in this case provided by the large top mass. At first sight this idea looks great: no fundamental scalars, no new states. But, looking closely, the advantages are largely illusory. First, in the SM the required value of $m_{t}$ is too large $m_{t} \geq 220 \mathrm{GeV}$ or so. Also a tremendous fine tuning is required, because $m_{t}$ would naturally be of the order of $M_{G U T}$ or $M_{P l}$ if no new physics is present (the hierarchy problem in a different form!). Supersymmetry could come to the rescue in this case also. In a minimal SUSY version the required value of the top mass is lowered [22i], $m_{t} \sim 205 \sin \beta \mathrm{GeV}$. But the resulting theory is physically indistinguishable from the MSSM with small $\tan \beta$, at least at low energies $[2 \overline{3}]$. This is because a strongly coupled Higgs looks the same as a $t \bar{t}$ pair.

The hierarchy problem is certainly not the only conceptual problem of the SM. There are many more: the proliferation of parameters, the mysterious pattern of fermion masses and so on. But while most of these problems can be postponed to the final theory that will take over at very large energies, of order $M_{G U T}$ or $M_{P l}$, the hierarchy problem arises from the unstability of the low energy theory and requires a solution at relatively low energies. A supersymmetric extension of the SM provides a way out which is well defined, computable and that preserves all virtues of the SM. The necessary SUSY breaking can be introduced through soft terms that do not spoil the stability of scalar masses. Precisely those terms arise from supergravity when it is spontaneously broken in a hidden sector [24i] But alternative mechanisms of SUSY breaking are also being considered [25]. In the most familiar approach SUSY is broken in a hidden sector and the scale of SUSY breaking is very large of order $\Lambda \sim \sqrt{G_{F}^{-1 / 2} M_{P}}$ where $M_{P}$ is the Planck mass. But since the hidden sector only communicates with the visible sector through gravitational interactions the splitting of the SUSY multiplets is much smaller, in the TeV energy domain, and the Goldstino is practically decoupled. In an alternative scenario the (not so much) hidden sector is connected to the visible one by ordinary gauge interactions. As these are much stronger than the gravitational interactions, $\Lambda$ can be much smaller, as low as 10-100 TeV. It follows that the Goldstino is very light in these models (with mass of order or below $1 \mathrm{eV}$ typically) and is the lightest, stable SUSY particle, but its couplings are observably large. The radiative decay of the lightest neutralino into the Goldstino leads to detectable photons. The signature of photons comes out naturally in this SUSY breaking pattern: with respect to the MSSM, in the gauge mediated model there are typically more photons and less missing energy. Gravitational and gauge mediation are extreme alternatives: a spectrum of intermediate cases is conceivable. The main appeal of gauge mediated models is a better protection against flavour changing neutral currents. In the gravitational version even if we accept that gravity leads to degenerate scalar masses at a scale near $M_{P l}$ the running of the masses down to the weak scale can generate mixing induced by the large masses of the third generation fermions [2 $2 \overline{6}$ ].

\subsection{Hints from Experiment}

\subsubsection{Unification of Couplings}

At present the most direct phenomenological evidence in favour of supersymmetry is obtained from the unification of couplings in GUTs. Precise LEP data on $\alpha_{s}\left(m_{Z}\right)$ and $\sin ^{2} \theta_{W}$ confirm what was already known with less accuracy: standard one-scale GUTs fail in predicting $\sin ^{2} \theta_{W}$ given $\alpha_{s}\left(m_{Z}\right)$ (and $\alpha\left(m_{Z}\right)$ ) while SUSY GUTs [27i] are in agreement with the present, very precise, experimental results. According to the recent analysis of ref. $\left[2 \bar{Z}_{1}^{\prime}\right.$, if one starts from the known values of $\sin ^{2} \theta_{W}$ and $\alpha\left(m_{Z}\right)$, one finds for $\alpha_{s}\left(m_{Z}\right)$ the results:

$$
\begin{aligned}
& \alpha_{s}\left(m_{Z}\right)=0.073 \pm 0.002(\text { Standard GUTs }) \\
& \alpha_{s}\left(m_{Z}\right)=0.129 \pm 0.010(\text { SUSY GUTs })(6.1)
\end{aligned}
$$

to be compared with the world average experimental value $\alpha_{s}\left(m_{Z}\right)=0.119(4)$. 


\subsubsection{Dark Matter}

There is solid astrophysical and cosmological evidence $\left[22_{1}^{\prime}\right]$, [30 $\left.{ }^{\prime}\right]$ that most of the matter in the universe does not emit electromagnetic radiation, hence is "dark". Some of the dark matter must be baryonic but most of it must be non baryonic. Non baryonic dark matter can be cold or hot. Cold means non relativistic at freeze out, while hot is relativistic. There is general consensus that most of the non baryonic dark matter must be cold dark matter. A couple of years ago the most likely composition was quoted to be around $80 \%$ cold and $20 \%$ hot. At present it appears to me that the need of a sizeable hot dark matter component is more uncertain. In fact, recent experiments have indicated the presence of a previously disfavoured cosmological constant component in $\Omega=\Omega_{m}+\Omega_{\Lambda} \overline{\Sigma_{2}} \overline{\overline{9}} \overline{\mathrm{i}}$. Here $\Omega$ is the total matter-energy density in units of the critical density, $\Omega_{m}$ is the matter component (dominated by cold dark matter) and $\Omega_{\Lambda}$ is the cosmological component. Inflationary theories almost inevitably predict $\Omega=1$ which is consistent with present data. At present, still within large uncertainties, the approximate composition is indicated to be $\Omega_{m} \sim 0.4$ and $\Omega_{\Lambda} \sim 0.6$ (baryonic dark matter gives $\Omega_{b} \sim 0.05$ ).

The implications for particle physics is that certainly there must exist a source of cold dark matter. By far the most appealing candidate is the neutralino, the lowest supersymmetric particle, in general a superposition of photino, Z-ino and higgsinos. This is stable in supersymmetric models with $\mathrm{R}$ parity conservation, which are the most standard variety for this class of models (including the Minimal Supersymmetric Standard Model:MSSM). A neutralino with mass of order $100 \mathrm{GeV}$ would fit perfectly as a cold dark matter candidate. Another common candidate for cold dark matter is the axion, the elusive particle associated to a possible solution of the strong CP problem along the line of a spontaneously broken Peccei-Quinn symmetry. To my knowledge and taste this option is less plausible than the neutralino. One favours supersymmetry for very diverse conceptual and phenomenological reasons, as described in the previous sections, so that neu- tralinos are sort of standard by now. For hot dark matter, the self imposing candidates are neutrinos. If we demand a density fraction $\Omega_{\nu} \sim 0.1$ from neutrinos, then it turns out that the sum of stable neutrino masses should be around $5 \mathrm{eV}$.

\subsubsection{Baryogenesis}

Baryogenesis is interesting because it could occur at the weak scale [3$\overline{1}_{1}^{\prime}$ but not in the SM. For baryogenesis one needs the three famous Sakharov conditions [32i]: B violation, $\mathrm{CP}$ violation and no termal equilibrium. In principle these conditions could be verified in the SM. B is violated by instantons when $\mathrm{kT}$ is of the order of the weak scale (but B-L is conserved). CP is violated by the CKM phase and out of equilibrium conditions could be verified during the electroweak phase transition. So the conditions for baryogenesis appear superficially to be present for it to occur at the weak scale in the SM. However,

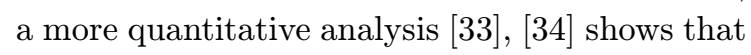
baryogenesis is not possible in the $\mathrm{SM}$ because there is not enough $\mathrm{CP}$ violation and the phase transition is not sufficiently strong first order, unless $m_{H}<80 \mathrm{GeV}$, which is by now excluded by LEP. Certainly baryogenesis could also occur below the GUT scale, after inflation. But only that part with $|B-L|>0$ would survive and not be erased at the weak scale by instanton effects. Thus baryogenesis at $k T \sim 10^{12}-10^{15} \mathrm{GeV}$ needs B-L violation at some stage like for $m_{\nu}$. The two effects could be related if baryogenesis arises from leptogenesis [35] then converted into baryogenesis by instantons. While baryogenesis at a large energy scale is thus not excluded it is interesting that recent studies have shown that baryogenesis at the weak scale could be possible in the MSSM [34]. In fact, in this model there are additional sources of CP violations and the bound on $m_{h}$ is modified by a sufficient amount by the presence of scalars with large couplings to the Higgs sector, typically the s-top. What is required is that $m_{h} \sim 80-110 \mathrm{GeV}$ (in the LEP2 range!), a s-top not heavier than the top quark and, preferentially, a small $\tan \beta$. 


\subsubsection{Neutrino Masses}

Recent data from Superkamiokande [36 MACRO [3글 imental basis for neutrino oscillations as an explanation of the atmospheric neutrino anomaly. In addition the solar neutrino deficit is also probably an indication of a different sort of neutrino oscillations. Results from the laboratory experiment by the LNSD collaboration [3효] can also be considered as a possible indication of yet another type of neutrino oscillation. But the preliminary data from Karmen [39 reproduce this evidence. The case of LNSD oscillations is far from closed but one can tentatively assume, pending the results of continuing experiments, that the signal will not persist. Then solar and atmospheric neutrino oscillations can possibly be explained in terms of the three known flavours of neutrinos without invoking extra sterile species. Neutrino oscillations for atmospheric neutrinos require $\nu_{\mu} \rightarrow \nu_{\tau}$ with $\Delta m_{\text {atm }}^{2} \sim 210^{-3} \mathrm{eV}^{2}$ and a nearly maximal mixing angle $\sin ^{2} 2 \theta_{a t m} \geq 0.8$. In most of the Superkamiokande allowed region the bound by Chooz [ī $\overline{0} \overline{0}]$ essentially excludes $\nu_{e} \rightarrow \nu_{\mu}$ oscillations for atmospheric neutrino oscillations. Furthermore the last results from Superkamiokande allow a solution of the solar neutrino deficit in terms of $\nu_{e}$ disappearance vacuum oscillations (as opposed to MSW [41] oscillations within the sun) with $\Delta m_{\text {sol }}^{2} \sim 10^{-10} \mathrm{eV}^{2}$ and again nearly maximal mixing angles. Among the large and small angle MSW solutions the small angle one is perhaps more likely at the moment (with [42 $\Delta m_{\text {sol }}^{2} \sim 0.510^{-5} \mathrm{eV}^{2}$ and $\left.\sin ^{2} 2 \theta_{\text {sol }} \sim 5.510^{-3}\right)$ than the large angle MSW solution. Of course experimental uncertainties are still large and the numbers given here are presumably only indicative. But by now it is very unlikely that all this evidence for neutrino oscillations will disappear or be explained away by astrophysics or other solutions. The consequence is that we have a substantial evidence that neutrinos are massive.

In a strict minimal standard model point of view neutrino masses could vanish if no right handed neutrinos existed (no Dirac mass) and lepton number was conserved (no Majorana mass).
In Grand Unified Theories both these assumptions are violated. The right handed neutrino is required in all unifying groups larger than $\mathrm{SU}(5)$. In $\mathrm{SO}(10)$ the 16 fermion fields in each family, including the right handed neutrino, exactly fit into the 16 dimensional representation of this group. This is really telling us that there is something in $\mathrm{SO}(10)$ ! The $\mathrm{SU}(5)$ alternative in terms of $\overline{5}+10$, without a right handed neutrino, is certainly less elegant. The breaking of $|B-L|, \mathrm{B}$ and $\mathrm{L}$ is also a generic feature of Grand Unification. In fact, the see-saw mechanism [433] explains the smallness of neutrino masses in terms of the large mass scale where $|B-L|$ and $L$ are violated. Thus, neutrino masses, as would be proton decay, are important as a probe into the physics at the GUT scale.

Oscillations only determine squared mass differences and not masses. The case of three nearly degenerate neutrinos is the only one that could in principle accomodate neutrinos as hot dark matter together with solar and atmospheric neutrino oscillations. According to our previous discussion, the common mass should be around 1$3 \mathrm{eV}$. The solar frequency could be given by a small 1-2 splitting, while the atmospheric frequency could be given by a still small but much larger 1,2-3 splitting. A strong constraint arises in the degenerate case from neutrinoless double beta decay which requires that the ee entry of $m_{\nu}$ must obey $\left|\left(m_{\nu}\right)_{11}\right| \leq 0.46 \mathrm{eV}$. As observed in ref. [44]"], this bound can only be satisfied if double maximal mixing is realized, i.e. if also solar neutrino oscillations occur with nearly maximal mixing. We have mentioned that it is not at all clear at the moment that a hot dark matter component is really needed [29 $\left.{ }^{2}\right]$. However the only reason to consider the fully degenerate solution is that it is compatible with hot dark matter. Note that for degenerate masses with $m \sim 1-3 \mathrm{eV}$ we need a relative splitting $\Delta m / m \sim \Delta m_{a t m}^{2} / 2 m^{2} \sim 10^{-3}-10^{-4}$ and an even smaller one for solar neutrinos. It is difficult to imagine a natural mechanism compatible with unification and the see-saw mechanism to arrange such a precise near symmetry.

If neutrino masses are smaller than for cosmological relevance, we can have the hierarchies 
$\left|m_{3}\right|>>\left|m_{2,1}\right|$ or $\left|m_{1}\right| \sim\left|m_{2}\right|>>\left|m_{3}\right|$. Note that we are assuming only two frequencies, given by $\Delta_{\text {sun }} \propto m_{2}^{2}-m_{1}^{2}$ and $\Delta_{a t m} \propto m_{3}^{2}-m_{1,2}^{2}$. We prefer the first case, because for quarks and leptons one mass eigenvalue, the third generation one, is largely dominant. Thus the dominance of $m_{3}$ for neutrinos corresponds to what we observe for the other fermions. In this case, $m_{3}$ is determined by the atmospheric neutrino oscillation frequency to be around $m_{3} \sim 0.05 \mathrm{eV}$. By the see-saw mechanism $m_{3}$ is related to some large mass $\mathrm{M}$, by $m_{3} \sim m^{2} / M$. If we identify $\mathrm{m}$ with either the Higgs vacuum expectation value or the top mass (which are of the same order), as suggested for third generation neutrinos by Grand Unification in simple $\mathrm{SO}(10)$ models, then M turns out to be around $M \sim 10^{15} \mathrm{GeV}$, which is consistent with the connection with GUT's. If solar neutrino oscillations are determined by vacuum oscillations, then $m_{2} \sim 10^{-5} \mathrm{eV}$ and we have that the ratio $m_{2} / m_{3}$ is well consistent with $\left(m_{c} / m_{t}\right)^{2}$.

A lot of attention is being devoted to the problem of a natural explanation of the observed nearly maximal mixing angle for atmospheric neutrino oscillations and possibly also for solar neutrino oscillations, if explained by vacuum oscillations [45i]. Large mixing angles are somewhat unexpected because the observed quark mixings are small and the quark, charged lepton and neutrino mass matrices are to some extent related in GUT's. There must be some special interplay between the neutrino Dirac and Majorana matrices in the see-saw mechanism in order to generate maximal mixing. It is hoped that looking for a natural explanation of large neutrino mixings can lead us to decripting some interesting message on the physics at the GUT scale.

\section{Comparing the Data with the Minimal Supersymmetric Standard Model}

The MSSM [200 is a completely specified, consistent and computable theory. There are too many parameters to attempt a direct fit of the data to the most general framework. So we consider two significant limiting cases: the "heavy" and the "light" MSSM.

The "heavy" limit corresponds to all s-particles being sufficiently massive, still within the limits of a natural explanation of the weak scale of mass. In this limit a very important result holds [46] : for what concerns the precision electroweak tests, the MSSM predictions tend to reproduce the results of the SM with a light Higgs, say $m_{H} \sim 100 \mathrm{GeV}$. So if the masses of SUSY partners are pushed at sufficiently large values the same quality of fit as for the SM is guaranteed. Note that for $m_{t} \sim 175 \mathrm{GeV}$ and $m_{H} \sim 70 \mathrm{GeV}$ the values of the four epsilons computed in the SM lead to a fit of the corresponding experimental values with $\chi^{2} \sim 4$, which is reasonable for d.o.f $=4$. This value corresponds to the fact that the central values of $\epsilon_{1}, \epsilon_{2}, \epsilon_{3}$ and $-\epsilon_{b}$ are all below the SM value by about $1 \sigma$, as can be seen from Fig. 4.2 .

In the "light" MSSM option some of the superpartners have a relatively small mass, close to their experimental lower bounds. In this case the pattern of radiative corrections may sizeably deviate from that of the SM [50 largest effects occur in vacuum polarisation amplitudes and/or the $Z \rightarrow b \bar{b}$ vertex. In particular we recall the following contributions :

i) a threshold effect in the $\mathrm{Z}$ wave function renormalisation [46-1] mostly due to the vector coupling of charginos and (off-diagonal) neutralinos to the $\mathrm{Z}$ itself. Defining the vacuum polarisation functions by $\Pi_{\mu \nu}\left(q^{2}\right)=-i g_{\mu \nu}\left[A(0)+q^{2} F\left(q^{2}\right)\right]+$ $q_{\mu} q_{\nu}$ terms, this is a positive contribution to $\epsilon_{5}=$ $m_{Z}^{2} F_{Z Z}^{\prime}\left(m_{Z}^{2}\right)$,the prime denoting a derivative with respect to $q^{2}$ (i.e. a contribution to a higher derivative term not included in the usual epsilon formalism). The $\epsilon_{5}$ correction shifts $\epsilon_{1}, \epsilon_{2}$ and $\epsilon_{3}$ by $-\epsilon_{5},-c^{2} \epsilon_{5}$ and $-c^{2} \epsilon_{5}$ respectively, where $c^{2}=$ $\cos ^{2} \theta_{W}$, so that all of them are reduced by a comparable amount. Correspondingly all the $\mathrm{Z}$ widths are reduced without affecting the asymmetries. This effect falls down particularly fast when the lightest chargino mass increases from a value close to $m_{Z} / 2$. Now that we know, from the LEP2 runs, that the chargino mass is not smaller than $m_{Z}$ its possible impact is drastically 
reduced.

ii) a positive contribution to $\epsilon_{1}$ from the virtual exchange of split multiplets of SUSY partners, for example of the scalar top and bottom superpartners [는 the top-bottom left-handed quark doublet. From the experimental value of $m_{t}$ not much space is left for this possibility, and the experimental value of $\epsilon_{1}$ is an important constraint on the spectrum. This is especially true now that the rather large lower limits on the chargino mass reduce the size of a possible compensation from $\epsilon_{5}$.For example, if the stop is light then it must be mainly a right-handed stop. Also large values of $\tan \beta$ are disfavoured because they tend to enhance the splittings among SUSY partner multiplets. In general it is simpler to decrease the predicted values of $\epsilon_{2}$ and $\epsilon_{3}$ by taking advantage of $\epsilon_{5}$ than to decrease $\epsilon_{1}$, because the negative shift from $\epsilon_{5}$ is most often counterbalanced by the increase from the effect of split SUSY multiplets.

iii) a negative contribution to $\epsilon_{b}$ due to the virtual exchange of a charged Higgs [4 $\left.4 \overline{8}^{\prime}\right]$. If one defines, as customary, $\tan \beta=v_{2} / v_{1}$ ( $v_{1}$ and $v_{2}$ being the vacuum expectation values of the Higgs doublets giving masses to the down and up quarks, respectively), then, for negligible bottom Yukawa coupling or $\tan \beta<<m_{t} / m_{b}$, this contribution is proportional to $m_{t}^{2} / \tan ^{2} \beta$.

iv) a positive contribution to $\epsilon_{b}$ due to virtual chargino-s-top exchange [499'] which in this case is proportional to $m_{t}^{2} / \sin ^{2} \beta$ and prefers small $\tan \beta$. This effect again requires the chargino and the s-top to be light in order to be sizeable.

With the recent limits set by LEP2 on the masses of SUSY partners the above effects are small enough that other contributions from vertex diagrams could be comparable. Thus in the following we will only consider the experimental values of the epsilons obtained at the level denoted by "All Asymmetries" which only assumes lepton universality.

We have analysed the problem of what configurations of masses in the "light" MSSM are

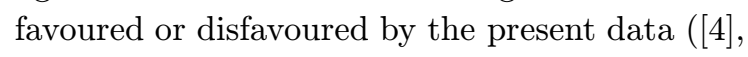

updating ref. $\left.\left[51_{1}^{1}\right]\right)$. We find that no lower limits on the masses of SUSY partners are obtained which are better than the direct limits. One exception is the case of s-top and s-bottom masses, which are severely constrained by the $\epsilon_{1}$ value and also, at small $\tan \beta$, by the increase at LEP2 of the direct limit on the Higgs mass. Charged higgs masses are also rather severely constrained. Since the central values of $\epsilon_{1}, \epsilon_{2}$ and $\epsilon_{3}$ are all below the SM it is convenient to make $\epsilon_{5}$ as large as possible. For this purpose light gaugino and s-lepton masses are favoured. We find that for $m_{\chi_{1}^{+}} \sim 90-120 \mathrm{GeV}$ the effect is still sizeable. Also favoured are small values of $\tan \beta$ that allow to put s-lepton masses relatively low, say, in the range 100-500 GeV, without making the split in the isospin doublets too large for $\epsilon_{1}$. Charged Higgses must be heavy because they contribute to $\epsilon_{b}$ with the wrong sign. A light right-handed stop could help on $R_{b}$ for a higgsino-like chargino. But one needs small mixing (the right-handed stop must be close to the mass eigenstate) and beware of the higgs mass constraint at small $\tan \beta$ (a higgs mass above $83 \mathrm{GeV}$, the range of LEP2 at $\sqrt{s}=183 \mathrm{GeV}$, starts being a strong constraint at small $\tan \beta$ ). So we prefer in the following to keep the s-top mass large. The limits on $b \rightarrow s \gamma$ also prefer heavy charged higgs and s-top [52].

\section{The LEP2 Programme and the Search for the Higgs and New Physics}

The LEP2 programme has started in the second part of 1996. At first the energy has been fixed at $161 \mathrm{GeV}$, which is the most favourable energy for the measurement of $m_{W}$ from the cross-section for $e^{+} e^{-} \rightarrow W^{+} W^{-}$at threshold. Then gradually the energy was brought up to $172,183,189$ $\mathrm{GeV}$. It will be increased up to a maximum of about $200 \mathrm{GeV}$ to be reached in mid '99. An integrated luminosity of about $150 \mathrm{pb}^{-1}$ per year is now achievable (in fact more was achieved in 1998). LEP2 has been approved to run until the end of 2000, before the shutdown for the installation of the LHC. The main goals of LEP2 are 
the search for the Higgs and for new particles, the measurement of $m_{W}$ and the investigation of the triple gauge vertices $W W Z$ and $W W \gamma$. A complete updated survey of the LEP2 physics is collected in the two volumes of ref. [53in.

An important competitor of LEP2 is the Tevatron collider. In mid 2000 the Tevatron will start RunII with the purpose of collecting a few $\mathrm{fb}^{-1}$ of integrated luminosity at $2 \mathrm{TeV}$. The competition is especially on the search of new particles, but also on $m_{W}$ and the triple gauge vertices. For example, for supersymmetry while the Tevatron is superior for gluinos and squarks, LEP2 is strong on Higgses, charginos, neutralinos and sleptons. There are plans for RunIII to start in 2002 or so with the purpose of collecting of the order $5 \mathrm{fb}^{-1}$ of integrated luminosity per year. Then the Tevatron could also hope to find the Higgs before the LHC starts if the Higgs mass is close to the LEP2 range.

Concerning the Higgs it is interesting to recall that the large value of $m_{t}$ has important implications on $m_{H}$ both in the minimal SM [54 $]$ [56.] and in its minimal supersymmetric extension [57 $m_{H}$ that follow from the observed value of $m_{t}$.

It is well known $[\overline{5} \overline{4}]-[5 \overline{6}]$ that in the SM with only one Higgs doublet a lower limit on $m_{H}$ can be derived from the requirement of vacuum stability. The limit is a function of $m_{t}$ and of the energy scale $\Lambda$ where the model breaks down and new physics appears. Similarly an upper bound on $m_{H}$ (with mild dependence on $m_{t}$ ) is obtained [60 1] from the requirement that up to the scale $\Lambda$ no Landau pole appears. The lower limit on $m_{H}$ is particularly important in view of the search for the Higgs at LEP2. Indeed the issue is whether one can reach the conclusion that if a Higgs is found at LEP2, i.e. with $m_{H} \leq m_{Z}$, then the SM must break down at some scale $\Lambda>1 \mathrm{TeV}$.

The possible instability of the Higgs potential $V[\phi]$ is generated by the quantum loop corrections to the classical expression of $V[\phi]$. At large $\phi$ the derivative $V^{\prime}[\phi]$ could become negative and the potential would become unbound from below. The one-loop corrections to $V[\phi]$ in the SM are well known and change the dominant term at large $\phi$ according to $\lambda \phi^{4} \rightarrow(\lambda+$ $\left.\gamma \log \phi^{2} / \Lambda^{2}\right) \phi^{4}$. The one-loop approximation is not enough for our purposes, because it fails at large enough $\phi$, when $\gamma \log \phi^{2} / \Lambda^{2}$ becomes of order 1 . The renormalization group improved version of the corrected potential leads to the replacement $\lambda \phi^{4} \rightarrow \lambda(\Lambda) \phi^{\prime 4}(\Lambda)$ where $\lambda(\Lambda)$ is the running coupling and $\phi^{\prime}(\mu)=\exp \int^{t} \gamma\left(t^{\prime}\right) d t^{\prime} \phi$, with $\gamma(t)$ being an anomalous dimension function and $t=\log \Lambda / v$ ( $v$ is the vacuum expectation value $\left.v=\left(2 \sqrt{2} G_{F}\right)^{-1 / 2}\right)$. As a result, the positivity condition for the potential amounts to the requirement that the running coupling $\lambda(\Lambda)$ never becomes negative. A more precise calculation, which also takes into account the quadratic term in the potential, confirms that the requirements of positive $\lambda(\Lambda)$ leads to the correct bound down to scales $\Lambda$ as low as $\sim 1 \mathrm{TeV}$. The running of $\lambda(\Lambda)$ at one loop is given by:

$$
\frac{d \lambda}{d t}=\frac{3}{4 \pi^{2}}\left[\lambda^{2}+3 \lambda h_{t}^{2}-9 h_{t}^{4}+\text { gauge terms }\right],
$$

with the normalization such that at $t=0, \lambda=$ $\lambda_{0}=m_{H}^{2} / 2 v^{2}$ and the top Yukawa coupling $h_{t}^{0}=$ $m_{t} / v$. We see that, for $m_{H}$ small and $m_{t}$ large, $\lambda$ decreases with $t$ and can become negative. If one requires that $\lambda$ remains positive up to $\Lambda=10^{15}$ $10^{19} \mathrm{GeV}$, then the resulting bound on $m_{H}$ in the SM with only one Higgs doublet is given by [5ㄴㄷㄴ.

$m_{H}>134+2.1\left[m_{t}-173.8\right]-4.5 \frac{\alpha_{s}\left(m_{Z}\right)-0.119}{0.006}$.

Summarizing, we see that the discovery of a Higgs particle at LEP2, or $m_{H} \lesssim 100 \mathrm{GeV}$, would imply that the SM breaks down at a scale $\Lambda$ of the order of a few TeV. It can be shown [54] that the lower limit is not much relaxed even if strict vacuum stability is replaced by some sufficiently long metastability.

The upper limit on the Higgs mass in the SM is important for assessing the chances of success of the LHC as an accelerator designed to solve the Higgs problem. The upper limit [60, $\left[0_{1}^{\prime}\right]$ arises from the requirement that the Landau pole associated with the non asymptotically free behaviour of the $\lambda \phi^{4}$ theory does not occur below the scale 
$\Lambda$. The initial value of $\lambda$ at the weak scale increases with $m_{H}$ and the derivative is positive at large $m_{H}$. Thus if $m_{H}$ is too large the Landau pole occurs at too low an energy. The upper limit on $m_{H}$ has been recently reevaluated ['599']. For $m_{t} \sim 175 \mathrm{GeV}$ one finds $m_{H} \lesssim 180 \mathrm{GeV}$ for $\Lambda \sim M_{G U T}-M_{P l}$ and $m_{H} \lesssim 0.5-0.8 \mathrm{TeV}$ for $\Lambda \sim 1 \mathrm{TeV}$. Actually, for $m_{t} \sim 174 \mathrm{GeV}$, only a small range of values for $m_{H}$ is allowed, $130<m_{H}<\sim 200 \mathrm{GeV}$, if the SM holds up to $\Lambda \sim M_{G U T}$ or $M_{P l}$.

A particularly important example of theory where the above bounds do not apply and in particular the lower bound is violated is the MSSM, which we now discuss. As is well known [200], in the MSSM there are two Higgs doublets, which implies three neutral physical Higgs particles and a pair of charged Higgses. The lightest neutral Higgs, called $h$, should be lighter than $m_{Z}$ at tree-level approximation. However, radiative corrections [ $\left[6 \hat{1}_{1}^{\prime}\right]$ increase the $h$ mass by a term proportional to $m_{t}^{4}$ and logarithmically dependent on the stop mass . Once the radiative corrections are taken into account the $h$ mass still remains rather small: for $m_{t}=174 \mathrm{GeV}$ one finds the limit (for all values of $\operatorname{tg} \beta$ ) $m_{h}<130 \mathrm{GeV}$ [5현]. Actually there are reasons to expect that $m_{h}$ is well below the bound. In fact, if $h_{t}$ is large at the GUT scale, which is suggested by the large observed value ot $m_{t}$ and by a natural onsetting of the electroweak symmetry breaking induced by $m_{t}$, then at low energy a fixed point is reached in the evolution of $m_{t}$. The fixed point corresponds to $m_{t} \sim 205 \sin \beta \mathrm{GeV}$ (a good approximate relation for $\left.\operatorname{tg} \beta=v_{\text {up }} / v_{\text {down }}<10\right)$. If the fixed point situation is realized, then $m_{h}$ is considerably below the bound, as shown in ref. [58

In conclusion, for $m_{t} \sim 174 \mathrm{GeV}$, we have seen that, on the one hand, if a Higgs is found at LEP the SM cannot be valid up to $M_{P l}$. On the other hand, if a Higgs is found at LEP, then the MSSM has good chances, because this model would be excluded for $m_{h}>130 \mathrm{GeV}$.

For the SM Higgs, which plays the role of a benchmark also important for a more general context, the LEP2 reach has been studied in detail [53]. At $200 \mathrm{GeV}$ with about $150 \mathrm{pb}^{-1}$ per experiment one can discover or exclude a SM Higgs up to about $105 \mathrm{GeV}$ of mass. In the MSSM a more complicated discussion is needed because there are several Higgses and the parameter space is multidimensional. Only the lightest MSSM Higgs $h$ is accessible at LEP2. The dominant production processes are $e^{+} e^{-}>h Z$ and $e^{+} e^{-}->h A$, where $\mathrm{A}$ is the CP odd MSSM Higgs particle. They are nicely complementary. At given $m_{h}$ within the range of interest, at large $\tan \beta$ the first process is the relevant one, while the second determines the bound at small $\tan \beta$. The absolute lower limit on $m_{h}$ for a given beam energy and integrated luminosity is always below the limit on the SM Higgs, because the crossections are smaller. For example the present limit is around $90 \mathrm{GeV}$ for the SM Higgs and around $80 \mathrm{GeV}$ for the MSSM Higgs. It is interesting that by the end of LEP2 one will have completely explored the region at small $\tan \beta$ (below a value of about 5), which is a particularly likely region.

A main goal of LEP2 is the search for supersymmetry. For charginos the discovery range at LEP2 is only limited by the beam energy for practically all values of the parameters. In fact the typical limit is at present about $90 \mathrm{GeV}$. Thus every increase of the beam energy is directly translated into the upper limit in chargino mass for discovery or exclusion. For the Tevatron the discovery range is much more dependent on the position in parameter space. For some limited regions of this space, with $1 \mathrm{fb}^{-1}$ of integrated luminosity, the discovery range for charginos at the Tevatron goes well beyond $m_{\chi}=$ $100 \mathrm{GeV}$, i.e. the boundary of LEP2, but in much of the parameter space LEP2 at the maximum energy would be sensitive to larger chargino masses.

The stop is probably the lightest squark. For a light stop the most likely decay modes are $\tilde{t} \rightarrow$ $b \chi^{+}$if kinematically allowed, otherwise $\tilde{t} \rightarrow c \chi$. At LEP2 the discovery range is up to about $\left(E_{\text {beam }}-10\right) \mathrm{GeV}$. At the Tevatron there is some difference between the two possible decay modes and some dependence on the position in the $\tilde{t}-\chi$ or the $\tilde{t}-\chi^{+}$planes, but in general the Tevatron is very powerful for s-quarks and gluinos and 
much of the LEP2 range is already excluded by the Tevatron.

By now most of the discovery potential of LEP2 for supersymmetry has been already deployed. For example, the limit on the chargino mass was about $45 \mathrm{GeV}$ after LEP1 and is now about $90 \mathrm{GeV}$ and can only improve up to $100 \mathrm{GeV}$. For the Higgs the experimental task is more demanding and so one is only a bit more than half way through: the lower limit on the SM Higgs was around $67 \mathrm{GeV}$ after LEP1, is now about $90 \mathrm{GeV}$ and could go up to $105 \mathrm{GeV}$ or so. So there are still fair chances for LEP2 to find the Higgs, especially because the attainable range of masses is particularly likely in the MSSM.

The study of the $W^{+} W^{-}$crosssection is a very important chapter of LEP2 physics [5 $5 \overline{3}$. In the Born approximation three Feynman diagrams contribute to the crosssection, as shown in Fig. $10_{-}^{\prime \prime}$ In the two s-channel exchange diagrams the triple gauge vertices $W W \gamma$ and $W W Z$ appear, while the third is the t-channel neutrino exchange that only involves well established charged current couplings. One loop radiative corrections have also been computed. It is interesting that if we take neutrino exchange alone or neutrino plus $\gamma$ exchanges alone the crosssection increases much faster with energy than the complete result. This corresponds to the good convergence properties of the SM which in fact is renormalisable. Indeed, the WW crosssection is related to the imaginary part of the WW loop contribution to the amplitude for $e^{+} e^{-} \rightarrow e^{+} e^{-}$. The good large energy behavior of the former crosssection is related to the convergence ot the latter loop correction. The data neatly confirm the SM prediction as shown in Fig. ${ }_{1}^{1} \overline{1}_{\mathbf{r}}^{1}$ Thus the WW crosssection supports the specific form of the triple gauge vertices as predicted by the SM. More detailed studies with large statistics are useful to set bounds on possible departures from the exact SM predicted couplings. In fact the study of triple gauge vertices is another major task of LEP2. The capabilities of LEP2 in this domain are comparable to those of the LHC. LEP2 can push down the existing direct limits considerably down. For given anomalous couplings the de-

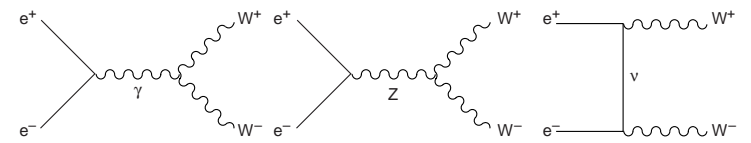

Figure 10: Lowest order Feynman diagrams for $e^{+} e^{-} \rightarrow W^{+} W^{-}$.

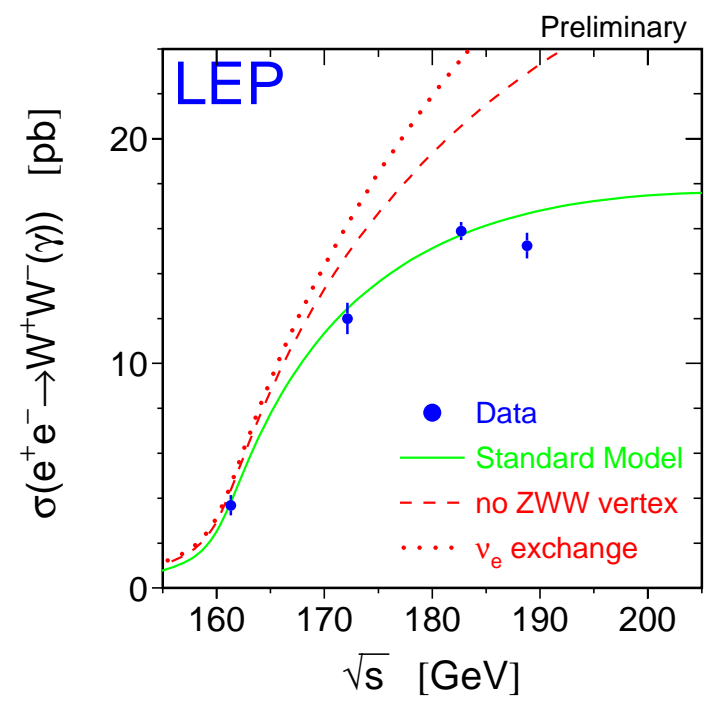

Figure 11: Data vs theory for the WW cross-section measured at LEP2. The solid line is the SM prediction. The dashed and dotted lines refer to only a subset diagrams as indicated.

partures from the SM are expected to increase with energy. For the energy and the luminosity available at LEP2, given the accuracy of the SM established at LEP1, it is however not very likely, to find signals of new physics in the triple gauge vertices. The measurement of $m_{W}$ is been done at LEP2 from the $W^{+} W^{-}$cross-section at threshold and from direct reconstruction of the $\mathrm{W}$ mass from the final state after $\mathrm{W}$ decay. At present $m_{W}$ is known with an error of $\pm 60 \mathrm{MeV}$ from the combined LEP2 and Tevatron direct measurements (see table 1), with the same error of $\pm 90 \mathrm{MeV}$ at LEP2 and at the Tevatron. From the fit to all electroweak data one finds $m_{W}=80370 \pm 27 \mathrm{MeV}$ (see eq.(13.10 $\left.\overline{1}_{1}^{\prime}\right)$ ), in agreement with the direct measurement. As a consequence the goal for LEP2 is to measure $m_{W}$ with an accuracy $\delta m_{W} \leq \pm(30-40) \mathrm{MeV}$, in order to provide an additional significant check of the 
theory.

For the threshold method [53] the minimum of the statistical error is obtained for $\sqrt{s}=2 m_{W}+$ $0.5 \mathrm{GeV}=161 \mathrm{GeV}$, which in fact was the initial operating energy of LEP2. At threshold the WW crossesection is dominated by the neutrino t-channel exchange which is quite model independent. The total error of this method is dominated by the statistics. With the collected luminosity at $161 \mathrm{GeV}$ of $\sim 10 p b^{-1}$ per experiment, the present combined result is $m_{W}=(80.4 \pm 0.2 \pm$ $0.03) \mathrm{GeV}$ [3] threshold this method is not sufficient by itself.

In principle the direct reconstruction method can use the totally hadronic or the semileptonic final states $e^{+} e^{-} \rightarrow W^{+} W^{-} \rightarrow j j j j$ or $j j l \nu$. The total branching ratio of the hadronic modes is $49 \%$, while that of the $\ell=e, \mu$ semileptonic channels is $28 \%$. The hadronic channel has more statistics but could be severely affected by nonperturbative strong interaction effects: colour recombination among the jets from different W's and Bose correlations among mesons in the final state from WW overlap. Colour recombination is perturbatively small. But gluons with $E<\Gamma_{W}$ are important and non-perturbative effects could be relatively large, of the order of $10-100 \mathrm{MeV}$. Similarly for Bose correlations. One is not in a position to really quantify the associated uncertainties. Fortunately the direct reconstruction from the semi-leptonic channels can, by itself, lead to a total error $\delta m_{W}= \pm 44 \mathrm{MeV}$, for the combined four experiments, each with $500 \mathrm{pb}^{-1}$ of luminosity collected at $\sqrt{s} \geq 175 \mathrm{GeV}$. Thus the goal of measuring $m_{W}$ with an accuracy below $\delta m_{W}= \pm 50 \mathrm{MeV}$ can be fulfilled, and it is possible to do better by learning from the data how to limit the error from colour recombination and Bose correlations.

\section{Conclusion}

Today in particle physics we follow a double approach: from above and from below. From above there are, on the theory side, quantum gravity (that is superstrings), GUT theories and cosmo- logical scenarios. On the experimental side there are underground experiments (e.g. searches for neutrino oscillations and proton decay), cosmic ray observations, satellite experiments (like COBE, IRAS etc) and so on. From below, the main objectives of theory and experiment are the search of the Higgs and of signals of particles beyond the Standard Model (typically supersymmetric particles). Another important direction of research is aimed at the exploration of the flavour problem: study of $\mathrm{CP}$ violation and rare decays. The general expectation is that new physics is close by and that should be found very soon if not for the complexity of the necessary experimental technology that makes the involved time scale painfully long.

\section{References}

[1] For reviews of radiative corrections for LEP1 physics, see, for example:

G. Altarelli, R. Kleiss and C. Verzegnassi (eds.), Z Physics at LEP1 (CERN 89-08, Geneva, 1989), Vols. 1-3;

Precision Calculations for the $\mathrm{Z}$ Resonance, ed. by D. Bardin, W. Hollik and G. Passarino, CERN Rep. 95-03 (1995);

M.I. Vysotskii, V.A. Novikov, L.B. Okun and A.N. Rozanov, hep-ph/9606253 or Phys.Usp. 39, 503 (1996).

[2] See, for example:

D. Karlen, Proceedings of ICHEP,98, Vancouver, 1998.

[3] The LEP Elelectroweak Working Group, LEPEWWG/98-01, (1998).

[4] For a complete list of references see (which sections $4-6$ are an update of):

G. Altarelli, R. Barbieri and F. Caravaglios, Int. J. Mod. Phys. A13, 1031 (1998).

[5] F. Jegerlehner, Z.Phys. C32, 195 (1986); B.W. Lynn, G. Penso and C. Verzegnassi, Phys. Rev. D35, 42 (1987);

H. Burkhardt et al., Z.Phys. C43, 497 (1989); F. Jegerlehner, Progr. Part. Nucl. Phys. 27, 32 (1991);

M.L. Swartz, Preprint SLAC-PUB-6710 (1994);

M.L. Swartz, Phys. Rev. D53, 5268 (1996);

A.D. Martin and D. Zeppenfeld, Phys.Lett. B345, 558 (1995); 
R.B. Nevzorov, A.V. Novikov and M.I. Vysotskii, hep-ph/9405390;

H. Burkhardt and B. Pietrzyk, Phys. Lett. B356, 398 (1995);

S. Eidelman and F. Jegerlehner, Z.Phys. C67, 585 (1995);

R. Alemany, M. Davier and A. Hocker, Europ. Phys. J. C2, 123 (1998).

[6] S. Groote et al., hep-ph/9802374;

M. Davier and A. Hocker, Phys. Lett. B419, 419 (1998);

J.H. Kuhn and M. Steinhauser, hep-ph/9802241;

J. Erler, hep-ph/9803453;

M. Davier and A. Hocker, hep-ph/9805470.

[7] See, for example:

Y. Dokshitser, Proceedings of ICHEP,98, Vancouver, 1998.

[8] G. Degrassi, P. Gambino and A. Vicini, Phys. Lett. B383, 219 (1996);

G. Degrassi, P. Gambino and A. Sirlin, Phys. Lett. B394, 188 (1997);

G. Degrassi, P. Gambino, M. Passera and A. Sirlin, hep-ph/9708311.

[9] CHARM Collaboration, J.V. Allaby et al., Phys. Lett. B177, 446 (1986); Z. Phys. C36, 611 (1987);

CDHS Collaboration, H. Abramowicz et al., Phys. Rev. Lett. 57, 298 (1986);

A. Blondel et al., Z. Phys. C45, 361 (1990;

CCFR Collaboration, K. McFarland, hepex/9701010.

[10] NuTeV Collaboration, K.S.McFarland et al., hep-ex/9806013.

[11] C.S. Wood et al., Science 2751759 (1997).

[12] CHARM II Collaboration, P. Vilain et al., Phys. Lett. B335, 246 (1997).

[13] P. Gambino and A. Sirlin, Phys. Rev. Lett. 73, 621 (1994);

S.Dittmaier et al., Nucl. Phys. B426, 249 (1994);

S. Dittmaier, D. Schildknecht and G. Weiglein, Nucl. Phys. B465, 3 (1996).

[14] L. Alvarez-Gaumé, J. Polchinski and M. Wise, Nucl. Phys. B221, 495 (1983);

R. Barbieri and L. Maiani, Nucl. Phys. B224, 32 (1983).

[15] M. Veltman, Nucl. Phys. B123, 589 (1977); S. Bertolini and A. Sirlin, Nucl. Phys. B248, 589 (1984).
[16] For a recent review, see R.S. Chivukula, hep$\mathrm{ph} / 9803219$.

[17] Particle Data Group, The Europ. Phys. J. C3, 1 (1998).

[18] G.G.Ross, "Grand Unified Theories", Benjamin, 1985;

R.N. Mohapatra, "Unification and Supersymmetry" Springer-Verlag, 1986.

[19] E. Gildener, Phys. Rev. D14, 1667 (1976);

E. Gildener and S. Weinberg, Phys. Rev. D15, 3333 (1976).

[20] H.P. Nilles, Phys. Rep. C110, 1 (1984);

H.E. Haber and G.L. Kane, Phys. Rep. C117, 75 (1985);

R. Barbieri, Riv. Nuovo Cim. 11, 1 (1988).

[21] For a review, see, for example, C.T. Hill in Perspectives on Higgs Physics, ed. G. Kane, World Scientific, Singapore, 1993, and references therein.

[22] W.A. Bardeen, T.E. Clark and S.T. Love, Phys. Lett. B237, 235 (1990);

M. Carena et al., Nucl. Phys. B369, 33 (1992).

[23] A. Hasenfratz et al., UCSD/PTH 91-06(1991).

[24] A. Chamseddine, R. Arnowitt and P. Nath, Phys. Rev. Lett. 49, 970 (1982);

R. Barbieri, S. Ferrara and C. Savoy, Phys. Lett. 110B, 343 (1982);

E. Cremmer et al., Phys. Lett. 116B, 215 (1982).

[25] For a review, see G.F.Giudice and R.Rattazzi, hep-ph/9801271 and references therein.

[26] A.Nelson, Proceedings of ICHEP'98, Vancouver, 1998.

[27] S. Dimopoulos, S. Raby and F. Wilczek, Phys.Rev. D24, 1681 (1981); S. Dimopoulos and H. Georgi, Nucl. Phys.B193, 150 (1981) 1 ;

L.E. Ibáñez and G.G. Ross, Phys. Lett. 105B, 439 (1981).

[28] P. Langacker and N.Polonsky, hep-ph/9503214.

[29] R. Kolb, Proceedings of ICHEP'98, Vancouver, 1998.

[30] M. Spiro, Proceedings of ICHEP'98, Vancouver, 1998.

[31] V.A. Kuzmin, V.A. Rubakov and M.E. Shaposhnikov,Phys. Lett. 155B, 36 (1985); M.E.Shaposhnikov,Nucl. Phys.B287, 757 (1987); Nucl. Phys.B299, 797 (1988). 
[32] A.D. Sakharov, JETP Lett. 91B, 24 (1967).

[33] A.G. Cohen, D.B. Kaplan and A.E. Nelson, Annu. Rev. Part. Sci. 43, 27 (1993); M. Quirós, Helv. Phys. Acta 67, 451 (1994); V.A. Rubakov and M.E. Shaposhnikov, hep$\mathrm{ph} / 9603208$.

[34] M. Carena, M. Quirós, and C.E.M. Wagner, Nucl. Phys. B524, 3 (1998).

[35] See, for example, M. Fukugita and T.Yanagida, Phys. Lett. B174, 45 (1986);

G. Lazarides and Q.Shafi, Phys. Lett. B258, 305(1991).

[36] Y. Fukuda et al., hep-ex/9805006, hepex/9805021 and hep-ph/9807003.

[37] M. Ambrosio et al., hep-ex/9807005.

[38] C. Athanassopoulos et al., Phys. Rev. Lett. 77, 3082 (1996), nucl-ex/9706006 and nuclex/9709006.

[39] B. Armbruster et al., Phys. Rev. C57, 3414 (1998) and G. Drexlin, talk at Wein'98.

[40] M. Apollonio et al., Phys. Lett. B420, 397 (1998).

[41] L. Wolfenstein, Phys. Rev. D17, 2369 (1978); S.P. Mikheyev and A. Yu Smirnov, Sov. J. Nucl. Phys. 42, 913 (1986).

[42] G.L. Fogli, E. Lisi and D. Montanino, hepph/9709473;

J.N. Bahcall, P.I. Krastev and A. Yu Smirnov, hep-ph/9807216;

R. Barbieri, L.J. Hall, D. Smith, A. Strumia and N. Weiner, hep/ph 9807235.

[43] M. Gell-Mann, P. Ramond and R. Slansky, in Supergravity, ed. by D.Freedman et al, North Holland, 1979;

T.Yanagida, Prog. Theo. Phys. B135, 66 (1978).

[44] F. Vissani, hep-ph/9708483;

H. Georgi and S.L. Glashow, hep-ph/9808293.

[45] For an introduction to this problem and list of references, see for example, G. Altarelli and F. Feruglio, hep-ph/9807353, hep-ph/9809596, hep-ph/9812475

[46] R. Barbieri, F. Caravaglios and M. Frigeni, Phys. Lett. B279, 169 (1992).

[47] R. Barbieri and L. Maiani, Nucl. Phys. B224, 32 (1983);

L. Alvarez-Gaumé, J. Polchinski and M. Wise, Nucl. Phys. B221, 495 (1983).
[48] W. Hollik, Mod. Phys. Lett. A5, 1909 (1990).

[49] A. Djouadi et al., Nucl. Phys. B349, 48 (1991); M. Boulware and D. Finell, Phys. Rev. D44, 2054 (1991). The sign discrepancy between these two papers appears now to be solved in favour of the second one.

[50] See, e.g., S.Pokorski,Proceedings of ICHEP'96, Warsaw, 1996.

See also, P.H. Chankowski, J. Ellis, S. Pokorski, Phys. Lett. B423, 327 (1998).

[51] G. Altarelli, R. Barbieri and F. Caravaglios, Phys. Lett. B314, 357 (1993).

[52] A. Brignole, F. Feruglio and F. Zwirner, $Z$. Phys. C71, 679 (1996).

[53] G. Altarelli, T. Sjöstrand and F. Zwirner (eds.), "Physics at LEP2", CERN Report 9503.

[54] M. Sher, Phys. Rep. 179, 273 (1989); Phys. Lett. B317, 159 (1993).

[55] G. Altarelli and G. Isidori, Phys. Lett. B337, 141 (1994).

[56] J.A. Casas, J.R. Espinosa and M. Quirós, Phys. Lett. B342, 171 (1995).

[57] J.A. Casas et al., Nucl. Phys. B436, 3 (1995); EB439, 466 (1995).

[58] M. Carena and C.E.M. Wagner, Nucl. Phys. B452, 45 (1995).

[59] T. Hambye and K. Riesselmann, Phys. Rev. D55, 7255 (1997).

[60] See, for example, M. Lindner, Z. Phys. 31, 295 (1986).

[61] H. Haber and R. Hempfling, Phys. Rev. Lett. 66, 1815 (1991);

J. Ellis, G. Ridolfi and F. Zwirner, Phys. Lett. B257, 83 (1991);

Y. Okado, M. Yamaguchi and T. Yanagida, Progr. Theor. Phys. Lett. 85, 1 (1991);

R. Barbieri, F. Caravaglios and M. Frigeni, Phys. Lett. B258, 167 (1991). For a 2-loop improvement, see also:

R. Memplfling and A.H. Hoang, Phys. Lett. B331, 99 (1994). 